\title{
Conformal Galilei algebras, symmetric polynomials and singular vectors
}

\author{
Libor Křžka, Petr Somberg
}

\begin{abstract}
We classify and explicitly describe homomorphisms of Verma modules for conformal Galilei algebras $\mathfrak{c g a}_{\ell}(d, \mathbb{C})$ with $d=1$ for any integer value $\ell \in \mathbb{N}$. The homomorphisms are uniquely determined by singular vectors as solutions of certain differential operators of flag type, and identified with specific polynomials arising as coefficients in the expansion of a parametric family of symmetric polynomials into power sum symmetric polynomials.
\end{abstract}

Keywords: Conformal Galilei algebra, Verma module, differential equation of flag type, symmetric polynomial.

2010 Mathematics Subject Classification: 53A30, 57S20, 20 B30.

\section{Contents}

Introduction 1

1 Geometric realization of induced modules 2

2 Conformal Galilei algebras $\mathfrak{c g a}_{\ell}(d, \mathbb{R})$ and their representation theory 7

2.1 Representation theoretical conventions .................. 8

2.2 Geometric realization of Verma modules . . . . . . . . . . . . . . . . . 9

3 Verma modules for conformal Galilei algebras 14

3.1 Singular vectors and generating series . . . . . . . . . . . . 16

3.2 Singular vectors and differential equations of flag type $\ldots \ldots \ldots \ldots \ldots$

Appendix A Symmetric polynomials and functions

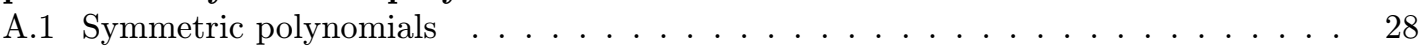

A.2 Newton's identities . . . . . . . . . . . . . . . . . . . . . 29

A.3 The Cauchy kernel . . . . . . . . . . . . . . . . . . . . . 31

\section{Introduction}

An important structure associated to a system of partial differential equations on manifolds is its algebra of symmetries. In the case when such a system is of geometric origin, this algebra allows to extract many fundamental properties of the former system (e.g. generates from a given solution of the system other solutions) and has a neat and delicate relationship to various properties of the underlying manifold.

As for the perspective of their applications in theoretical physics, one of the most important examples of such a system of partial differential equations is the free Schrödinger equation. A classical result says that its symmetry algebra is recognized as the Schrödinger algebra, which is a basic representative of the family of the so called conformal Galilei algebras, see [6]. The real conformal Galilei algebras $\mathfrak{c g a}_{\ell}(d, \mathbb{R})$ parameterized by $d \in \mathbb{N}$ (called the spatial dimension) and $\ell \in \frac{1}{2} \mathbb{N}_{0}$ (called the spin), are (generalizations of) non-relativistic analogues of Lie algebras 
$\mathfrak{s o}(d+1,2, \mathbb{R})$ acting by conformal transformations on $\mathbb{R}^{d, 1}$ and constructed as their contractions. In contrast with conformal Lie algebras, the conformal Galilei algebras are not semisimple. There are several topics of recent interest related to conformal Galilei algebras, e.g. the classical mechanics with higher order time derivatives, the non-relativistic AdS/CFT correspondence for conformal Galilei algebras related to the flat holography in the limit when the radius of the AdS spacetime tends to infinity.

A standard mathematical setup consists in the change of the previous perspective. Having the symmetry realized by a conformal Galilei algebra, we may ask for the classification of its invariants. In the present article, we consider the pair consisting of a conformal Galilei algebra $\mathfrak{c} \mathfrak{a q}_{\ell}(d, \mathbb{C})$ for $d=1$ with any integer spin $\ell \in \mathbb{N}_{0}$ and its standard Borel subalgebra, and analyze the composition structure of Verma modules induced from characters of the standard Borel subalgebra. Homomorphisms of Verma modules are determined by the highest/lowest weight (or singular) vectors, which have another important significance. Namely, they are in a bijection with invariant differential operators acting on smooth sections of principal series representations supported on the homogeneous space for the conformal Galilei group and induced from the dual character to that used for Verma modules. Although our considerations on the homogeneous space for conformal Galilei group are purely local, on a general smooth manifold plays the role of the transitive action of conformal Galilei group the Newton-Cartan geometric structure.

Based on the techniques of geometric representation theory and harmonic analysis, the main achievement in the present article is the complete classification and precise positions of singular vectors in Verma modules for conformal Galilei algebras $\mathfrak{c g a}_{\ell}(1, \mathbb{C})$ with $\ell \in \mathbb{N}_{0}$, thereby completing several partial and non-complete results in this direction we are aware of, see [2], 3], 4], 1]. The complete classification of singular vectors in Verma modules for conformal Galilei algebras $\mathfrak{c} \mathfrak{g a} \mathfrak{a}_{\ell}(1, \mathbb{C})$ with $\ell \in \frac{1}{2}+\mathbb{N}_{0}$ is given in [4]. The starting point leading to our results is based on the reformulation of the former task to a problem of classifying polynomial solutions of certain differential equations of flag type. We found it quite convenient and rather indispensable to express all solutions of this equation in terms of certain polynomials organized into finite products of generating formal power series. Let us note that a classification of all finite weight modules over the conformal Galilei algebras $\mathfrak{c g a}_{\ell}(1, \mathbb{C})$ with any $\ell \in \frac{1}{2} \mathbb{N}_{0}$ has been done in [10].

Let us briefly summarize the content of our article. In Section 1, we describe the geometric realization of induced modules on the homogeneous space for a general complex finite-dimensional Lie group and its Borel subgroup. In Section 2, we discuss representation theoretical properties of conformal Galilei algebras in the spatial dimension $d=1$ together with a class of lowest weight modules. Namely, we describe $\mathcal{D}$-module realization of these modules supported on the closed (point) orbit for the Borel subgroup. In fact, they are realized as the dual spaces of jets of sections of line bundles supported at the unit coset of the homogeneous space for conformal Galilei group. The complete classification together with explicit formulas for singular vectors is achieved by passing to the Fourier dual picture, see Section 3 . This converts the former problem to describe the solution space of the differential equation of flag type. The rest of this section is then devoted to an explicit description of its solution space through the technique of generating formal power series and their products. This is quite convenient and also surprising way due to a canonical multiplicative structure on the solution space. For the reader's convenience, we remind in Appendix $\mathrm{A}$ a few basic formulas related to symmetric polynomials which are needed in the discussion. We close the article by highlighting several open questions and problems related to our work.

Throughout the article, we use the standard notation $\mathbb{Z}, \mathbb{N}$ and $\mathbb{N}_{0}$ for the set of integers, the set of natural numbers and the set of natural numbers including zero, respectively.

\section{Geometric realization of induced modules}

In this section we describe a geometric realization of Verma modules for finite-dimensional complex Lie algebras, which are not necessarily complex semisimple Lie algebras.

Let $G$ be a complex finite-dimensional Lie group with its Lie algebra $\mathfrak{g}$. Furthermore, let $\mathfrak{h}$ be a 
Cartan subalgebra of $\mathfrak{g}$, which means that $\mathfrak{h}$ is a nilpotent Lie subalgebra of $\mathfrak{g}$ such that $\mathfrak{n}_{\mathfrak{g}}(\mathfrak{h})=\mathfrak{h}$, where $\mathfrak{n}_{\mathfrak{g}}(\mathfrak{h})$ denotes the normalizer of $\mathfrak{h}$ in $\mathfrak{g}$. Let us note that there exists a Cartan subalgebra of $\mathfrak{g}$ and that two Cartan subalgebras of $\mathfrak{g}$ are conjugate by an automorphism of $\mathfrak{g}$, and in particular they are isomorphic. If we define a generalized eigenspace $\mathfrak{g}_{\alpha} \subset \mathfrak{g}$ for $\alpha \in \mathfrak{h}^{*}$ by

$$
\mathfrak{g}_{\alpha}=\left\{X \in \mathfrak{g} ;(\operatorname{ad}(H)-\alpha(H))^{k} X=0 \text { for all } H \in \mathfrak{h} \text { and } k \gg 0\right\},
$$

then we may introduce the notion of root spaces, roots, positive and negative roots for the Lie algebra $\mathfrak{g}$ as in the case of semisimple Lie algebras. We denote by $\Delta$ the root system of $\mathfrak{g}$ with respect to $\mathfrak{h}$ and by $\Delta^{+}$and $\Delta^{-}$a positive and negative root subspaces in $\Delta$, respectively.

We associate to the positive and negative root system $\Delta^{+}$and $\Delta^{-}$the nilpotent Lie subalgebras

$$
\mathfrak{n}=\bigoplus_{\alpha \in \Delta^{+}} \mathfrak{g}_{\alpha} \quad \text { and } \quad \overline{\mathfrak{n}}=\bigoplus_{\alpha \in \Delta^{-}} \mathfrak{g}_{\alpha}
$$

and solvable Lie subalgebras

$$
\mathfrak{b}=\mathfrak{h} \oplus \mathfrak{n} \quad \text { and } \quad \overline{\mathfrak{b}}=\mathfrak{h} \oplus \overline{\mathfrak{n}}
$$

of $\mathfrak{g}$, respectively. Moreover, we get a triangular decomposition

$$
\mathfrak{g}=\overline{\mathfrak{n}} \oplus \mathfrak{h} \oplus \mathfrak{n}
$$

of the Lie algebra $\mathfrak{g}$. We refer the reader to 7 for more detailed exposition of Cartan subalgebras of general Lie algebras. Let us note that the Lie subalgebras $\mathfrak{b}$ and $\overline{\mathfrak{b}}$ are not in general Borel subalgebras in the sense of maximal solvable Lie subalgebras of $\mathfrak{g}$. However, we will call $\mathfrak{b}$ and $\overline{\mathfrak{b}}$ the standard and opposite standard Borel subalgebra of $\mathfrak{g}$, respectively, because of the analogy with the triangular decomposition of semisimple Lie algebras.

Since $\mathfrak{n}_{\mathfrak{g}}(\mathfrak{b})=\mathfrak{b}$, we define the Borel subgroup $B$ of $G$ with its Lie algebra $\mathfrak{b}$ as the connected component of the identity of the closed subgroup $N_{G}(\mathfrak{b})$ of $G$. Then $p: G \rightarrow G / B$ is a principal $B$-bundle and $X=G / B$ is the corresponding flag manifold for $G$. We define the closed subgroup $\bar{N}$ of $G$ to be the image of $\overline{\mathfrak{n}}$ under the exponential mapping exp: $\mathfrak{g} \rightarrow G$, so that the mapping

$$
\exp : \overline{\mathfrak{n}} \rightarrow \bar{N}
$$

is a diffeomorphism. Therefore, for $g \in G$ the mapping $f_{g}: \bar{N} \rightarrow X$ given by

$$
f_{g}(n)=p(g n)=g n B
$$

for $n \in \bar{N}$ is an open embedding, and the manifold $X$ is covered by open subsets $U_{g}=p(g \bar{N})$ for which we have

$$
X=\bigcup_{g \in G} U_{g}
$$

Hence, we obtain the atlas $\left\{\left(U_{g}, u_{g}\right)\right\}_{g \in G}$ on $X$, where $u_{g}: U_{g} \rightarrow \overline{\mathfrak{n}}$ is defined by

$$
u_{g}^{-1}=f_{g} \circ \exp
$$

Furthermore, let $s_{g}: U_{g} \rightarrow G$ be a local section of the principal $B$-bundle $p: G \rightarrow X$ defined by

$$
s_{g}(x)=g f_{g}^{-1}(x)=g s_{e}\left(g^{-1} \cdot x\right)
$$

for $x \in U_{g}$ and $g \in G$. The local section $s_{g}: U_{g} \rightarrow G$ gives a trivialization of the principal $B$-bundle $p: G \rightarrow X$ over $U_{g}$, i.e. $p^{-1}\left(U_{g}\right) \simeq U_{g} \times B$ as principal $B$-bundles over $U_{g}$.

For $\lambda \in \operatorname{Hom}_{B}(\mathfrak{b}, \mathbb{C})$ there exists a $G$-equivariant sheaf of rings of twisted differential operators $\mathcal{D}_{X}(\lambda)$ on $X$ such that $\mathcal{D}_{X}(0) \simeq \mathcal{D}_{X}$, where $\mathcal{D}_{X}$ is the sheaf of rings of differential operators 
on $X$. If we denote by $\mathcal{D}_{X}(\lambda)^{\text {op }}$ the sheaf of rings opposite to $\mathcal{D}_{X}(\lambda)$, then $\mathcal{D}_{X}(\lambda)^{\text {op }}$ is also a $G$-equivariant sheaf of rings of twisted differential operators on $X$, and we have

$$
\mathcal{D}_{X}(\lambda)^{\mathrm{op}} \simeq \mathcal{D}_{X}(2 \rho-\lambda),
$$

where the character $\rho \in \operatorname{Hom}_{B}(\mathfrak{b}, \mathbb{C})$ of $\mathfrak{b}$ is defined by

$$
\rho(a)=-\frac{1}{2} \operatorname{tr}_{\mathfrak{g} / \mathfrak{b}} \operatorname{ad}(a)
$$

for $a \in \mathfrak{b}$. Since $\mathcal{D}_{X}(\lambda)$ is a $G$-equivariant sheaf of rings of twisted differential operators on $X$, we obtain a Lie algebra homomorphism

$$
\alpha_{\mathcal{D}_{X}(\lambda)}: \mathfrak{g} \rightarrow \Gamma\left(X, \mathcal{D}_{X}(\lambda)\right) .
$$

Now, let us consider a left $\mathcal{D}_{X}(\lambda)$-module $\mathcal{M}$. Then the vector space of global sections $\Gamma(X, \mathcal{M})$ is a left $\Gamma\left(X, \mathcal{D}_{X}(\lambda)\right)$-module. Taking into account (1.12), we get a $\mathfrak{g}$-module structure on $\Gamma(X, \mathcal{M})$. A local section $s_{g}: U_{g} \rightarrow G$ gives an isomorphism

$$
j_{s_{g}}^{\lambda}:\left.\left.\mathcal{D}_{X}(\lambda)\right|_{U_{g}} \stackrel{\sim}{\rightarrow} \mathcal{D}_{X}\right|_{U_{g}}
$$

of sheaves of rings of twisted differential operators on $U_{g}$. Hence, we define

$$
\pi_{\lambda}^{g}(a)=\left(j_{s_{g}}^{\lambda} \circ \alpha_{\mathcal{D}_{X}(\lambda)}\right)(a)
$$

for $a \in \mathfrak{g}$.

Let $\left\{f_{1}, f_{2}, \ldots, f_{\operatorname{dim} \overline{\mathfrak{n}}}\right\}$ be a basis of $\overline{\mathfrak{n}}$. Then

$$
u_{g}(x)=\sum_{i=1}^{\operatorname{dim} \overline{\mathfrak{n}}} u_{g}^{i}(x) f_{i}, \quad x \in U_{g},
$$

where the functions $u_{g}^{i}: U_{g} \rightarrow \mathbb{C}$ are called the coordinate functions on $U_{g}$.

Theorem 1.1. Let $\lambda \in \operatorname{Hom}_{B}(\mathfrak{b}, \mathbb{C})$. Then we have

$$
\begin{aligned}
& \pi_{\lambda}^{g}(a)=-\sum_{i=1}^{\operatorname{dim} \overline{\mathfrak{n}}}\left[\frac{\operatorname{ad}\left(u_{g}(x)\right) e^{\operatorname{ad}\left(u_{g}(x)\right)}}{e^{\operatorname{ad}\left(u_{g}(x)\right)}-\operatorname{id}_{\overline{\mathfrak{n}}}}\left(e^{-\operatorname{ad}\left(u_{g}(x)\right)} \operatorname{Ad}\left(g^{-1}\right) a\right)_{\overline{\mathfrak{n}}}\right]_{i} \partial_{u_{g}^{i}} \\
& +\lambda\left(\left(e^{-\operatorname{ad}\left(u_{g}(x)\right)} \operatorname{Ad}\left(g^{-1}\right) a\right)_{\mathfrak{b}}\right)
\end{aligned}
$$

for all $a \in \mathfrak{g}$, where $x \in U_{g}$ and $[b]_{i}$ denotes the $i$-th coordinate of $b \in \overline{\mathfrak{n}}$ with respect to the basis $\left\{f_{1}, f_{2}, \ldots, f_{\operatorname{dim} \overline{\mathfrak{n}}}\right\}$ of $\overline{\mathfrak{n}}$. In particular, we have

$$
\pi_{\lambda}^{e}(a)=-\sum_{i=1}^{\operatorname{dim} \overline{\mathfrak{n}}}\left[\frac{\operatorname{ad}\left(u_{e}(x)\right)}{e^{\operatorname{ad}\left(u_{e}(x)\right)}-\mathrm{id}_{\overline{\mathfrak{n}}}} a\right]_{i} \partial_{u_{e}^{i}} \quad \text { for } \quad a \in \overline{\mathfrak{n}}
$$

and

$$
\pi_{\lambda}^{e}(a)=\sum_{i=1}^{\operatorname{dim} \overline{\mathfrak{n}}}\left[\operatorname{ad}\left(u_{e}(x)\right) a\right]_{i} \partial_{u_{e}^{i}}+\lambda(a) \quad \text { for } \quad a \in \mathfrak{h} .
$$

Proof. The proof of the parallel claim for any finite-dimensional semisimple Lie algebra and its parabolic subalgebra was given in $[8$. However, the assumption of the semisimplicity of $\mathfrak{g}$ was redundant there, the only important structural data is a triangular decomposition of the Lie algebra in question. In particular, for the triangular decomposition (1.4) of our finite-dimensional (non-semisimple) Lie algebra $\mathfrak{g}$ the proof given in [8, Theorem 1.3] is applicable verbatim to the present case. 
Let $\lambda \in \operatorname{Hom}_{B}(\mathfrak{b}, \mathbb{C})$ be a character of $\mathfrak{b}$. Then we denote by $\mathbb{C}_{\lambda}$ the 1-dimensional representation of $\mathfrak{b}$ defined by

$$
a v=\lambda(a) v
$$

for $a \in \mathfrak{b}$ and $v \in \mathbb{C}_{\lambda} \simeq \mathbb{C}$ as vector spaces.

Definition 1.2. Let $\lambda \in \operatorname{Hom}_{B}(\mathfrak{b}, \mathbb{C})$ be a character of $\mathfrak{b}$. Then the Verma module is the induced module

$$
M_{\mathfrak{b}}^{\mathfrak{g}}(\lambda)=\operatorname{Ind}_{\mathfrak{b}}^{\mathfrak{g}} \mathbb{C}_{\lambda} \equiv U(\mathfrak{g}) \otimes_{U(\mathfrak{b})} \mathbb{C}_{\lambda} \simeq U(\overline{\mathfrak{n}}) \otimes_{\mathbb{C}} \mathbb{C}_{\lambda},
$$

where the last isomorphism of $U(\overline{\mathfrak{n}})$-modules follows from the Poincaré-Birkhoff-Witt (PBW for short) theorem.

Definition 1.3. Let $\lambda \in \operatorname{Hom}_{B}(\mathfrak{b}, \mathbb{C})$ be a character of $\mathfrak{b}$. Then a vector $v \in M_{\mathfrak{b}}^{\mathfrak{g}}(\lambda)$ is called a singular vector in the Verma module $M_{\mathfrak{b}}^{\mathfrak{g}}(\lambda)$ provided there exists a character $\mu \in \operatorname{Hom}_{B}(\mathfrak{b}, \mathbb{C})$ of $\mathfrak{b}$ such that $a v=\mu(a) v$ for all $a \in \mathfrak{b}$. We define a $\mathfrak{b}$-module

$$
M_{\mathfrak{b}}^{\mathfrak{g}}(\lambda)^{\mathfrak{b}}=\left\langle\left\{v \in M_{\mathfrak{b}}^{\mathfrak{g}}(\lambda) ; a v=\mu(a) v \text { for all } a \in \mathfrak{b} \text { and some } \mu \in \operatorname{Hom}_{B}(\mathfrak{b}, \mathbb{C})\right\}\right\rangle
$$

and call it the vector space of singular vectors. We observe that singular vector $v \in M_{\mathfrak{b}}^{\mathfrak{g}}(\lambda)$ with character $\mu \in \operatorname{Hom}_{B}(\mathfrak{b}, \mathbb{C})$ is also a weight vector with weight $\mu_{\mid \mathfrak{h}} \in \mathfrak{h}^{*}$.

Let us consider a homomorphism

$$
\varphi: M_{\mathfrak{b}}^{\mathfrak{g}}(\mu) \rightarrow M_{\mathfrak{b}}^{\mathfrak{g}}(\lambda)
$$

of Verma modules. This homomorphism of $\mathfrak{g}$-modules is uniquely determined by a homomorphism $\varphi_{0}: \mathbb{C}_{\mu} \rightarrow M_{\mathfrak{b}}^{\mathfrak{g}}(\lambda)$ of $\mathfrak{b}$-modules via the formula

$$
\varphi(a \otimes v)=a \varphi_{0}(v)
$$

for all $a \in U(\mathfrak{g})$ and $v \in \mathbb{C}_{\mu}$. However, since $a \varphi_{0}(v)=\mu(a) \varphi_{0}(v)$ for all $a \in \mathfrak{b}$ and $v \in \mathbb{C}_{\mu}$, we have $\varphi_{0}\left(\mathbb{C}_{\mu}\right) \subset M_{\mathfrak{b}}^{\mathfrak{g}}(\lambda)^{\mathfrak{b}}$. Therefore, we obtain a homomorphism

$$
\varphi_{0}: \mathbb{C}_{\mu} \rightarrow M_{\mathfrak{b}}^{\mathfrak{g}}(\lambda)^{\mathfrak{b}}
$$

of $\mathfrak{b}$-modules, which uniquely determines $\varphi$. In the opposite way, any such homomorphism $\varphi_{0}$ gives rise to a homomorphism $\varphi: M_{\mathfrak{b}}^{\mathfrak{g}}(\mu) \rightarrow M_{\mathfrak{b}}^{\mathfrak{g}}(\lambda)$ of Verma modules. Therefore, this gives us a vector space isomorphism

$$
\operatorname{Hom}_{\mathfrak{g}}\left(M_{\mathfrak{b}}^{\mathfrak{g}}(\mu), M_{\mathfrak{b}}^{\mathfrak{g}}(\lambda)\right) \simeq \operatorname{Hom}_{\mathfrak{b}}\left(\mathbb{C}_{\mu}, M_{\mathfrak{b}}^{\mathfrak{g}}(\lambda)^{\mathfrak{b}}\right) .
$$

Let $\left\{f_{1}, f_{2}, \ldots, f_{\operatorname{dim} \overline{\mathfrak{n}}}\right\}$ be a basis of $\overline{\mathfrak{n}}$. We denote by $\left\{x_{1}, x_{2}, \ldots, x_{\operatorname{dim} \overline{\mathfrak{n}}}\right\}$ the linear coordinate functions on $\overline{\mathfrak{n}}$ with respect to the given basis of $\overline{\mathfrak{n}}$ and by $\left\{y_{1}, y_{2}, \ldots, y_{\operatorname{dim}} \overline{\mathfrak{n}}\right\}$ the dual linear coordinate functions on $\overline{\mathfrak{n}}$. The Weyl algebra $\mathcal{A}_{\overline{\mathfrak{n}}}$ of the vector space $\overline{\mathfrak{n}}$ is generated by $\left\{x_{i}, \partial_{x_{i}} ; i=\right.$ $1,2, \ldots, \operatorname{dim} \overline{\mathfrak{n}}\}$ and the Weyl algebra $\mathcal{A}_{\overline{\mathfrak{n}}^{*}}$ of the vector space $\overline{\mathfrak{n}}^{*}$ by $\left\{y_{i}, \partial_{y_{i}} ; i=1,2, \ldots, \operatorname{dim} \overline{\mathfrak{n}}\right\}$. Furthermore, we define the algebraic Fourier transform

$$
\mathcal{F}: \mathcal{A}_{\overline{\mathfrak{n}}}^{\mathfrak{\sim}} \stackrel{\sim}{\longrightarrow} \mathcal{A}_{\mathfrak{\mathfrak { n }}^{*}}^{\mathfrak{g}}
$$

as an isomorphism of $\mathbb{C}$-algebras uniquely determined by

$$
\mathcal{F}\left(x_{i}\right)=-\partial_{y_{i}}, \quad \mathcal{F}\left(\partial_{x_{i}}\right)=y_{i}
$$

for all $i=1,2, \ldots, \operatorname{dim} \overline{\mathfrak{n}}$. We denote by $\mathcal{J}_{e}$ the left ideal of $\mathcal{A}_{\overline{\mathfrak{n}}}$ generated by polynomials on $\overline{\mathfrak{n}}$ vanishing at the point 0 . 
Since the mapping $u_{e}: U_{e} \rightarrow \overline{\mathfrak{n}}$ is a diffeomorphism, it induces an isomorphism

$$
\Psi_{u_{e}}: \mathcal{D}_{X}\left(U_{e}\right) \stackrel{\sim}{\longrightarrow} \Gamma\left(\overline{\mathfrak{n}}, \mathcal{D}_{\overline{\mathfrak{n}}}\right)
$$

of associative $\mathbb{C}$-algebras. Let us note that the Weyl algebra $\mathcal{A}_{\overline{\mathfrak{n}}}$ is contained in $\Gamma\left(\overline{\mathfrak{n}}, \mathcal{D}_{\overline{\mathfrak{n}}}\right)$. Moreover, we have

$$
\Psi_{u_{e}}\left(u_{e}^{i}\right)=x_{i} \quad \text { and } \quad \Psi_{u_{e}}\left(\partial_{u_{e}^{i}}\right)=\partial_{x_{i}}
$$

for $i=1,2, \ldots, \operatorname{dim} \overline{\mathfrak{n}}$, where the coordinate functions $\left\{u_{e}^{i} ; i=1,2, \ldots, \operatorname{dim} \overline{\mathfrak{n}}\right\}$ on $U_{e}$ are given by (1.15). By a completely analogous way as in $[8$, Section 2], we obtain an isomorphism of $\mathfrak{g}$-modules

$$
\Phi_{\lambda}: M_{\mathfrak{b}}^{\mathfrak{g}}(\lambda-\rho) \stackrel{\sim}{\longrightarrow} \mathcal{A}_{\mathfrak{\mathfrak { n }}}^{\mathfrak{g}} / \mathcal{J}_{e}
$$

uniquely determined by

$$
\Phi_{\lambda}\left(v_{\lambda-\rho}\right)=1 \bmod \mathcal{J}_{e},
$$

where $v_{\lambda-\rho}$ is the highest weight vector of $M_{\mathfrak{b}}^{\mathfrak{g}}(\lambda-\rho)$. The structure of a left $\mathfrak{g}$-module on $\mathcal{A}_{\mathfrak{n}} / \mathcal{J}_{e}$ is given through a homomorphism

$$
\pi_{\lambda}: \mathfrak{g} \rightarrow \mathcal{A}_{\overline{\mathfrak{n}}}^{\mathfrak{g}}
$$

of Lie algebras defined by

$$
\pi_{\lambda}=\Psi_{u_{e}} \circ \pi_{\lambda+\rho}^{e} .
$$

In the next theorem we realize the mapping $\Phi_{\lambda}$ in an explicit way. This result enables us to write singular vectors as elements of $M_{\mathfrak{b}}^{\mathfrak{g}}(\lambda-\rho) \simeq U(\overline{\mathfrak{n}}) \otimes_{\mathbb{C}} \mathbb{C}_{\lambda-\rho}$.

Theorem 1.4. Let $\left\{f_{1}, f_{2}, \ldots, f_{\operatorname{dim} \overline{\mathfrak{n}}}\right\}$ be a basis of $\overline{\mathfrak{n}}$ and $\left\{x_{1}, x_{2}, \ldots, x_{\operatorname{dim}} \overline{\mathfrak{n}}\right\}$ be the corresponding linear coordinate functions on $\overline{\mathfrak{n}}$. Further, let $\beta: S(\overline{\mathfrak{n}}) \rightarrow U(\overline{\mathfrak{n}})$ be the symmetrization map defined by

$$
\beta\left(a_{1} a_{2} \ldots a_{k}\right)=\frac{1}{k !} \sum_{\sigma \in S_{k}} a_{\sigma(1)} a_{\sigma(2)} \ldots a_{\sigma(k)}
$$

for all $k \in \mathbb{N}$ and $a_{1}, a_{2}, \ldots, a_{k} \in \overline{\mathfrak{n}}$. Then

$$
\Phi_{\lambda}\left(\beta\left(f_{i_{1}} f_{i_{2}} \ldots f_{i_{k}}\right) v_{\lambda-\rho}\right)=(-1)^{k} \partial_{x_{i_{1}}} \partial_{x_{i_{2}}} \ldots \partial_{x_{i_{k}}} \bmod \mathcal{J}_{e}
$$

for all $k \in \mathbb{N}$ and $i_{1}, i_{2}, \ldots, i_{k} \in\{1,2, \ldots, \operatorname{dim} \overline{\mathfrak{n}}\}$.

Proof. The proof goes along the same line as that in [8, Theorem 2.4].

The algebraic Fourier transform $\mathcal{F}: \mathcal{A}_{\overline{\mathfrak{n}}}^{\mathfrak{g}} \rightarrow \mathcal{A}_{\overline{\mathfrak{n}}^{*}}$ leads to an isomorphism

$$
\tau: \mathcal{A}_{\overline{\mathfrak{n}}}^{\mathfrak{g}} / \mathcal{J}_{e} \stackrel{\sim}{\longrightarrow} \mathcal{A}_{\overline{\mathfrak{n}}}{ }^{*} / \mathcal{F}\left(\mathcal{J}_{e}\right)
$$

of $\mathfrak{g}$-modules defined by

$$
Q \bmod \mathcal{J}_{e} \mapsto \mathcal{F}(Q) \bmod \mathcal{F}\left(\mathcal{J}_{e}\right)
$$

for all $Q \in \mathcal{A}_{\mathfrak{\mathfrak { n }}}$. The structure of a left $\mathfrak{g}$-module on $\mathcal{A}_{\mathfrak{\mathfrak { n }}^{*}} / \mathcal{F}\left(\mathcal{J}_{e}\right)$ is given through a homomorphism

$$
\hat{\pi}_{\lambda}: \mathfrak{g} \rightarrow \mathcal{A}_{\overline{\mathfrak{n}}^{*}}^{\mathfrak{g}}
$$

of Lie algebras, defined by

$$
\hat{\pi}_{\lambda}=\mathcal{F} \circ \pi_{\lambda}
$$


Since there is a canonical isomorphism of left $\mathcal{A}_{\overline{\mathfrak{n}}^{*}}{ }^{\mathfrak{g}}$-modules

$$
\mathbb{C}\left[\overline{\mathfrak{n}}^{*}\right] \stackrel{\sim}{\longrightarrow} \mathcal{A}_{\overline{\mathfrak{n}}^{*}}^{\mathfrak{g}} / \mathcal{F}\left(\mathcal{J}_{e}\right)
$$

we obtain immediately an isomorphism

$$
\tau \circ \Phi_{\lambda}: M_{\mathfrak{b}}^{\mathfrak{g}}(\lambda-\rho) \stackrel{\sim}{\longrightarrow} \mathbb{C}\left[\overline{\mathfrak{n}}^{*}\right]
$$

of $\mathfrak{g}$-modules. If we introduce a $\mathfrak{b}$-module

$$
\operatorname{Sol}\left(\mathfrak{g}, \mathfrak{b} ; \mathbb{C}\left[\overline{\mathfrak{n}}^{*}\right]\right)^{\mathcal{F}}=\left\{u \in \mathbb{C}\left[\overline{\mathfrak{n}}^{*}\right] ; \hat{\pi}_{\lambda}(a) u=\mu(a) u \text { for all } a \in \mathfrak{b} \text { and some } \mu \in \operatorname{Hom}_{B}(\mathfrak{b}, \mathbb{C})\right\},
$$

then by (1.41), we obtain an isomorphism of $\mathfrak{b}$-modules

$$
\tau \circ \Phi_{\lambda}: M_{\mathfrak{b}}^{\mathfrak{g}}(\lambda-\rho)^{\mathfrak{b}} \stackrel{\sim}{\longrightarrow} \operatorname{Sol}\left(\mathfrak{g}, \mathfrak{b} ; \mathbb{C}\left[\overline{\mathfrak{n}}^{*}\right]\right)^{\mathcal{F}} .
$$

Therefore, the algebraic problem of finding singular vectors in the Verma module $M_{\mathfrak{b}}^{\mathfrak{g}}(\lambda-\rho)$ is converted into an analytic problem of solving a system of partial differential equations on $\mathbb{C}\left[\overline{\mathfrak{n}}^{*}\right]$.

\section{Conformal Galilei algebras $\mathfrak{c g a}_{\ell}(d, \mathbb{R})$ and their represen- tation theory}

Conformal Galilei algebras $\mathfrak{c g a}_{\ell}(d, \mathbb{R})$ are generalized non-relativistic versions of conformal Lie algebras $\mathfrak{s o}(d+1,2, \mathbb{R})$ and parameterized by pairs $(d, \ell)$ with $d \in \mathbb{N}$ (called the spatial dimension) and $\ell \in \frac{1}{2} \mathbb{N}_{0}$ (called the spin). The Lie algebras $\mathfrak{c g a}_{\ell}(d, \mathbb{R})$ are not semisimple, and there exists a 1-dimensional central extension either for $d \in \mathbb{N}$ and $\ell$ half-integer or for $d=2$ and $\ell$ non-negative integer.

Let $\mathbb{R}^{1, d}$ be the $(d+1)$-dimensional space endowed with the canonical metric of signature $(1, d)$ and let $\left(t, x_{1}, x_{2}, \ldots, x_{d}\right)$ be the canonical linear coordinate functions on $\mathbb{R}^{1, d}$. The variable $t$ is referred to as the time coordinate while $x_{1}, x_{2}, \ldots, x_{d}$ are referred to as the spatial coordinates. Let us consider the infinitesimal transformations of $\mathbb{R}^{1, d}$ given by vector fields

$$
\begin{gathered}
H=\partial_{t}, \quad D=-2 t \partial_{t}-2 \ell \sum_{i=1}^{d} x_{i} \partial_{x_{i}}, \quad C=t^{2} \partial_{t}+2 \ell \sum_{i=1}^{d} t x_{i} \partial_{x_{i}} \\
M_{i j}=-x_{i} \partial_{x_{j}}+x_{j} \partial_{x_{i}}, \quad P_{n, i}=(-t)^{n} \partial_{x_{i}},
\end{gathered}
$$

where $i, j=1,2 \ldots, d$ and $n=0,1, \ldots, 2 \ell$ with $\ell \in \frac{1}{2} \mathbb{N}_{0}$. Their linear span has real dimension $\frac{d(d-1)}{2}+(2 \ell+1) d+3$ and is closed under the Lie bracket of vector fields. Consequently, we get finite-dimensional real Lie algebra with non-trivial Lie brackets

$$
\begin{gathered}
{[D, H]=2 H, \quad[C, H]=D, \quad[D, C]=-2 C,} \\
{\left[H, P_{n, i}\right]=-n P_{n-1, i}, \quad\left[D, P_{n, i}\right]=2(\ell-n) P_{n, i}, \quad\left[C, P_{n, i}\right]=(2 \ell-n) P_{n+1, i},} \\
{\left[M_{i j}, M_{k \ell}\right]=-\delta_{i, k} M_{j \ell}-\delta_{j, \ell} M_{i k}+\delta_{i, \ell} M_{j k}+\delta_{j, k} M_{i \ell},} \\
{\left[M_{i j}, P_{n, k}\right]=-\delta_{i, k} P_{n, j}+\delta_{j, k} P_{n, i},}
\end{gathered}
$$

where $i, j=1,2 \ldots, d$ and $n=0,1, \ldots, 2 \ell$. The Lie algebra $\mathfrak{c g a}_{\ell}(d, \mathbb{R})$ has a Lie subalgebra given by direct sum of $\mathfrak{s l}(2, \mathbb{R}) \simeq \mathfrak{s o}(2,1, \mathbb{R})$ generated by $C, D, H$ and $\mathfrak{s o}(d, \mathbb{R})$ generated by $M_{i j}$ for $i, j=1,2, \ldots, d$. The subspace generated by $P_{n, i}$ for $i=1,2, \ldots, d$ and $n=0,1, \ldots, 2 \ell$ forms an abelian ideal and we have $\mathfrak{c g a}_{\ell}(d, \mathbb{R}) \simeq(\mathfrak{s l}(2, \mathbb{R}) \oplus \mathfrak{s o}(d, \mathbb{R})) \ltimes \mathbb{V}_{2 \ell \omega, \omega_{1}}$, where $\mathbb{V}_{2 \ell \omega, \omega_{1}}$ is the irreducible finite-dimensional $(\mathfrak{s l}(2, \mathbb{R}) \oplus \mathfrak{s o}(d, \mathbb{R}))$-module with highest weight $\left(2 \ell \omega, \omega_{1}\right)$ (see the next section for the representation theoretical conventions concerning weights $\left.\omega, \omega_{1}\right)$.

The conformal Galilei algebras $\mathfrak{c g a}_{\ell}(d, \mathbb{R})$ admit two distinct types of central extensions according to the values of $d \in \mathbb{N}$ and $\ell \in \frac{1}{2} \mathbb{N}_{0}$. In particular, we have 
1) the mass central extension for $d \in \mathbb{N}$ and $\ell \in \frac{1}{2}+\mathbb{N}_{0}$,

$$
\left[P_{m, i}, P_{n, j}\right]=\delta_{i, j} \delta_{m+n, 2 \ell} I_{m} M, \quad I_{m}=(-1)^{m+\ell+\frac{1}{2}}(2 \ell-m) ! m !,
$$

2) the exotic central extension for $d=2$ and $\ell \in \mathbb{N}_{0}$,

$$
\left[P_{m, i}, P_{n, j}\right]=\varepsilon_{i, j} \delta_{m+n, 2 \ell} I_{m} \Theta, \quad I_{m}=(-1)^{m}(2 \ell-m) ! m !,
$$

where $\varepsilon_{1,2}=-\varepsilon_{2,1}=1$ and $\varepsilon_{1,1}=\varepsilon_{2,2}=0$.

Let us note that the generators of time translation $H$, space translations $P_{0, i}$, spatial rotations $M_{i j}$ and Galilei transformations $P_{1, i}$ form the Galilei algebra of $\mathbb{R}^{1, d}$.

In the rest of the article we shall restrict to the (complexification of the real) conformal Galilei algebras $\mathfrak{c g a}_{\ell}(d, \mathbb{C})$ for $d=1$ and $\ell \in \mathbb{N}$, and we denote $P_{n, 0}$ by $P_{n}$ for $n=0,1, \ldots, 2 \ell$. In particular, as follows from the previous paragraph there is no central extension in this case.

\subsection{Representation theoretical conventions}

The Lie algebra $\mathfrak{c g a}_{\ell}(1, \mathbb{C})$ is given by the complex vector space $\left\langle D, H, C, P_{0}, P_{1}, \ldots, P_{2 \ell}\right\rangle$ together with the following non-trivial Lie brackets

$$
\begin{array}{lll}
{[D, H]=2 H,} & {[C, H]=D,} & {[D, C]=-2 C,} \\
{\left[H, P_{n}\right]=-n P_{n-1},} & {\left[D, P_{n}\right]=2(\ell-n) P_{n},} & {\left[C, P_{n}\right]=(2 \ell-n) P_{n+1}}
\end{array}
$$

for all $n=0,1, \ldots, 2 \ell$.

Remark. If we denote

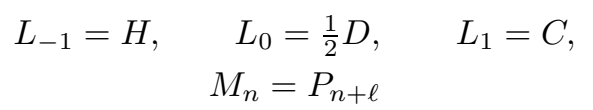

for $n=-\ell,-\ell+1, \ldots, \ell$, then we may write

$$
\left[L_{m}, L_{n}\right]=(m-n) L_{m+n}, \quad\left[L_{m}, M_{n}\right]=(\ell m-n) M_{m+n}, \quad\left[M_{m}, M_{n}\right]=0 .
$$

Now, for $\ell \in \mathbb{N}_{0}$ we may define an infinite-dimensional complex Lie algebra with a basis $L_{m}, M_{n}$ for $m, n \in \mathbb{Z}$ together with the Lie bracket given by (2.7) for all $m, n \in \mathbb{Z}$. This infinite-dimensional complex Lie algebra admits a universal central extension whose explicit form depends on the value of $\ell \in \mathbb{N}_{0}$. For $\ell=0$ we obtain the so called Heisenberg-Virasoro algebra and for $\ell=1$ we get the so called Bondi-Metzner-Sachs (BMS) algebra in dimension 3. This observation was the first evidence for the non-relativistic version of $\mathrm{AdS}_{3} / \mathrm{CFT}_{2}$ correspondence, see [5], 12].

Let us denote $\mathfrak{g}=\mathfrak{c g a}_{\ell}(1, \mathbb{C}), \ell \in \mathbb{N}$. Let $G$ be a connected complex Lie group with its Lie algebra $\mathfrak{g}$. By structural theory reviewed in Section 1, we choose the Cartan subalgebra $\mathfrak{h}$ of $\mathfrak{g}$ to be

$$
\mathfrak{h}=\mathbb{C} D \oplus \mathbb{C} P_{\ell}
$$

We define $\omega_{D}, \omega_{P_{\ell}} \in \mathfrak{h}^{*}$ by

$$
\omega_{D}(D)=1, \quad \omega_{D}\left(P_{\ell}\right)=0, \quad \omega_{P_{\ell}}(D)=0, \quad \omega_{P_{\ell}}\left(P_{\ell}\right)=1 .
$$

Then the root system of $\mathfrak{g}$ with respect to $\mathfrak{h}$ is $\Delta=\{ \pm k \alpha ; 1 \leq k \leq \ell\}, \alpha=2 \omega_{D}$, and the root spaces are

$$
\mathfrak{g}_{k \alpha}=\mathbb{C} P_{\ell-k}, \quad \mathfrak{g}_{\alpha}=\mathbb{C} H \oplus \mathbb{C} P_{\ell-1}, \quad \mathfrak{g}_{-\alpha}=\mathbb{C} C \oplus \mathbb{C} P_{\ell+1}, \quad \mathfrak{g}_{-k \alpha}=\mathbb{C} P_{\ell+k}
$$

for $k=2,3, \ldots, \ell$. Further, the positive root system is $\Delta^{+}=\{k \alpha ; 1 \leq k \leq \ell\}$ and the negative root system is $\Delta^{-}=\{-k \alpha ; 1 \leq k \leq \ell\}$. 
The Lie algebra $\mathfrak{g}$ is $|\ell|$-graded with respect to the grading given by $\mathfrak{g}_{i}=\mathfrak{g}_{i \alpha}$ for $0 \neq i \in \mathbb{Z}$ and $\mathfrak{g}_{0}=\mathfrak{h}$. We define nilpotent Lie subalgebras $\mathfrak{n}_{+}$and $\mathfrak{n}_{-}$of $\mathfrak{g}$ by

$$
\mathfrak{n}_{+}=\bigoplus_{i=1}^{\ell} \mathfrak{g}_{i}=\left\langle H, P_{0}, P_{1}, \ldots, P_{\ell-1}\right\rangle, \quad \mathfrak{n}_{-}=\bigoplus_{i=1}^{\ell} \mathfrak{g}_{-i}=\left\langle C, P_{\ell+1}, P_{\ell+2}, \ldots, P_{2 \ell}\right\rangle,
$$

and the standard Borel subalgebra $\mathfrak{b}$ of $\mathfrak{g}$ by

$$
\mathfrak{b}=\mathfrak{h} \oplus \mathfrak{n}_{-}=\left\langle D, C, P_{\ell}, P_{\ell+1}, \ldots, P_{2 \ell}\right\rangle .
$$

We remark that $\mathfrak{b}$ is contained in a maximal (standard) solvable Lie subalgebra of $\mathfrak{g}$, which is given by the linear span $\left\langle D, C, P_{0}, P_{1}, \ldots, P_{2 \ell}\right\rangle$. Moreover, we have a triangular decomposition

$$
\mathfrak{g}=\mathfrak{n}_{-} \oplus \mathfrak{h} \oplus \mathfrak{n}_{+}
$$

of the Lie algebra $\mathfrak{g}$ with $\left[\mathfrak{h}, \mathfrak{n}_{+}\right]=\mathfrak{n}_{+}$and $\left[\mathfrak{h}, \mathfrak{n}_{-}\right]=\mathfrak{n}_{-}$. The Borel subgroup $B$ of $G$ with its Lie algebra $\mathfrak{b}$ is defined by $B=N_{G}(\mathfrak{b})$.

Any character $\lambda \in \operatorname{Hom}_{B}(\mathfrak{b}, \mathbb{C})$ of $\mathfrak{b}$ is given by

$$
\lambda=\delta \widetilde{\omega}_{D}+p \widetilde{\omega}_{P_{\ell}}
$$

for some $\delta, p \in \mathbb{C}$, where $\widetilde{\omega}_{D}, \widetilde{\omega}_{P_{\ell}} \in \operatorname{Hom}_{B}(\mathfrak{b}, \mathbb{C})$ are equal to $\omega_{D}, \omega_{P_{\ell}} \in \mathfrak{h}^{*}$ regarded as trivially extended to $\mathfrak{b}=\mathfrak{h} \oplus \mathfrak{n}_{-}$. We denote the character $\delta \widetilde{\omega}_{D}+p \widetilde{\omega}_{P_{\ell}}$ for $\delta, p \in \mathbb{C}$ by $\lambda_{\delta, p}$. The character $\rho \in \operatorname{Hom}_{B}(\mathfrak{b}, \mathbb{C})$ of $\mathfrak{b}$ introduced in (1.11) is

$$
\rho=-\left(1+\frac{1}{2} \ell(\ell+1)\right) \widetilde{\omega}_{D}
$$

We shall denote

$$
\delta_{\rho}=-\left(1+\frac{1}{2} \ell(\ell+1)\right) \quad \text { and } \quad p_{\rho}=0 .
$$

Since for $\delta, p \in \mathbb{C}$ the character $\lambda_{\delta, p} \in \operatorname{Hom}_{B}(\mathfrak{b}, \mathbb{C})$ of $\mathfrak{b}$ defines the 1-dimensional representation $\mathbb{C}_{\delta, p}$ of $\mathfrak{b}$ through the formula (1.19), we associate to $\mathbb{C}_{\delta, p}$ the Verma module $M_{\ell}(\delta, p)$ :

$$
M_{\ell}(\delta, p)=U(\mathfrak{g}) \otimes_{U(\mathfrak{b})} \mathbb{C}_{\delta, p} \simeq U\left(\mathfrak{n}_{+}\right) \otimes_{\mathbb{C}} \mathbb{C}_{\delta, p},
$$

where the isomorphism of $U\left(\mathfrak{n}_{+}\right)$-modules follows from the PBW theorem. We denote by $v_{\delta, p}$ the lowest weight vector of $M_{\ell}(\delta, p)$ with lowest weight $\delta \omega_{D}+p \omega_{P_{\ell}}$. There is an automorphism $\omega: \mathfrak{g} \rightarrow \mathfrak{g}$ of $\mathfrak{g}$ defined by

$$
\omega(D)=-D, \quad \omega(H)=-C, \quad \omega(C)=-H, \quad \omega\left(P_{n}\right)=-P_{2 \ell-n}
$$

for $n=0,1, \ldots, 2 \ell$, called the Chevalley involution. Moreover, there exists a unique symmetric bilinear form $\langle\cdot, \cdot\rangle$ on $M_{\ell}(\delta, p)$, called the Shapovalov form, with its contravariance property $\left\langle v_{\delta, p}, v_{\delta, p}\right\rangle=1$ and $\langle a u, v\rangle=-\langle u, \omega(a) v\rangle$ for all $u, v \in M_{\ell}(\delta, p)$ and $a \in \mathfrak{g}$.

\subsection{Geometric realization of Verma modules}

Let us denote by $\left\{t, x_{0}, \ldots, x_{\ell-1}\right\}$ the linear coordinate functions on $\mathfrak{n}_{+}$with respect to the given basis $\left\{H, P_{0}, \ldots, P_{\ell-1}\right\}$ of $\mathfrak{n}_{+}$, and by $\left\{u, y_{0}, \ldots, y_{\ell-1}\right\}$ the dual linear coordinate functions on $\mathfrak{n}_{+}^{*}$. Then the Weyl algebra $\mathcal{A}_{\mathfrak{n}_{+}}^{\mathfrak{g}}$ is generated by

$$
\left\{t, x_{0}, \ldots, x_{\ell-1}, \partial_{t}, \partial_{x_{0}}, \ldots, \partial_{x_{\ell-1}}\right\}
$$

and the Weyl algebra $\mathcal{A}_{\mathfrak{n}_{+}^{*}}^{\mathfrak{g}}$ by

$$
\left\{u, y_{0}, \ldots, y_{\ell-1}, \partial_{u}, \partial_{y_{0}}, \ldots, \partial_{y_{\ell-1}}\right\} .
$$


Furthermore, the coordinate functions $\left\{u_{e}^{t}, u_{e}^{x_{0}}, \ldots, u_{e}^{x_{\ell-1}}\right\}$ on $U_{e}$ are defined by

$$
u_{e}(x)=u_{e}^{t}(x) H+\sum_{n=0}^{\ell-1} u_{e}^{x_{n}}(x) P_{n}
$$

for $x \in U_{e}$. Using (1.29), we obtain

$$
\Psi_{u_{e}}\left(u_{e}^{t}\right)=t, \quad \Psi_{u_{e}}\left(u_{e}^{x_{n}}\right)=x_{n}, \quad \Psi_{u_{e}}\left(\partial_{u_{e}^{t}}\right)=\partial_{t}, \quad \Psi_{u_{e}}\left(\partial_{u_{e}^{x_{n}}}\right)=\partial_{x_{n}}
$$

for $n=0,1, \ldots, \ell-1$. We define

$$
u(x)=t(x) H+\sum_{n=0}^{\ell-1} x_{n}(x) P_{n}
$$

for $x \in \mathfrak{n}_{+}$. Finally, the homomorphism $\pi_{\delta, p}: \mathfrak{g} \rightarrow \mathcal{A}_{\mathfrak{n}_{+}}^{\mathfrak{g}}$ of Lie algebras defined by (1.33) is

$$
\pi_{\delta, p}=\Psi_{u_{e}} \circ \pi_{\lambda_{\delta, p}+\rho}^{e}
$$

and its explicit form is a subject of the next theorem.

Let us recall that the Bernoulli polynomials $B_{n}(x)$ are defined by a generating function

$$
\frac{t e^{x t}}{e^{t}-1}=\sum_{n=0}^{\infty} B_{n}(x) \frac{t^{n}}{n !}
$$

for $0 \neq t \in \mathbb{R}$ and $x \in \mathbb{R}$. We denote $B_{n}=B_{n}(0)$ the first Bernoulli numbers.

Theorem 2.1. Let $\delta, p \in \mathbb{C}$. Then the embedding of $\mathfrak{g}$ into $\mathcal{A}_{\mathfrak{n}_{+}}^{\mathfrak{g}}$ and $\mathcal{A}_{\mathfrak{n}_{+}^{*}}^{\mathfrak{g}}$ is given by 1)

$$
\begin{gathered}
\pi_{\delta, p}(H)=-\partial_{t}-\sum_{j=1}^{\ell-1} \sum_{k=j}^{\ell-1}(-1)^{j-1} B_{j}\left(\begin{array}{c}
k \\
j
\end{array}\right) t^{j-1} x_{k} \partial_{x_{k-j}}, \\
\pi_{\delta, p}\left(P_{n}\right)=-\partial_{x_{n}}-\sum_{j=1}^{n}(-1)^{j} B_{j}\left(\begin{array}{c}
n \\
j
\end{array}\right) t^{j} \partial_{x_{n-j}}, \\
\hat{\pi}_{\delta, p}(H)=-u+\sum_{j=1}^{\ell-1} \sum_{k=j}^{\ell-1} B_{j}\left(\begin{array}{c}
k \\
j
\end{array}\right) y_{k-j} \partial_{y_{k}} \partial_{u}^{j-1}, \\
\hat{\pi}_{\delta, p}\left(P_{n}\right)=-y_{n}-\sum_{j=1}^{n} B_{j}\left(\begin{array}{c}
n \\
j
\end{array}\right) y_{n-j} \partial_{u}^{j}
\end{gathered}
$$

for $n=0,1, \ldots, \ell-1$;

2)

$$
\begin{aligned}
& \pi_{\delta, p}\left(P_{\ell}\right)=-\ell t \partial_{x_{\ell-1}}+p \\
& \pi_{\delta, p}(D)=-2 t \partial_{t}-2 \sum_{j=0}^{\ell-1}(\ell-j) x_{j} \partial_{x_{j}}+\delta+\delta_{\rho} \\
& \hat{\pi}_{\delta, p}\left(P_{\ell}\right)=\ell y_{\ell-1} \partial_{u}+p \\
& \hat{\pi}_{\delta, p}(D)=2 u \partial_{u}+2 \sum_{j=0}^{\ell-1}(\ell-j) y_{j} \partial_{y_{j}}+\delta-\delta_{\rho}
\end{aligned}
$$


3)

$$
\begin{aligned}
& \pi_{\delta, p}(C)=-t^{2} \partial_{t}-\sum_{j=0}^{\ell-1}(\ell-j) t x_{j} \partial_{x_{j}}-\sum_{j=0}^{\ell-2}(2 \ell-j) x_{j} \partial_{x_{j+1}}+\left(\delta+\delta_{\rho}\right) t \\
&+\sum_{j=1}^{\ell} B_{j}(\ell+1)\left(\begin{array}{l}
\ell \\
j
\end{array}\right) t^{j} x_{\ell-1} \partial_{x_{\ell-j}}+p(\ell+1) x_{\ell-1} \\
&+\sum_{j=2}^{\ell-1} \sum_{k=0}^{\ell-2} \frac{2(\ell-1-k)}{k+1} B_{j}\left(\begin{array}{c}
k+1 \\
j
\end{array}\right) t^{j} x_{k} \partial_{x_{k-j+1}}, \\
& \pi_{\delta, p}\left(P_{n}\right)=-\sum_{j=0}^{\ell-1} \sum_{k=n-\ell+1}^{n-j} B_{j}\left(\begin{array}{c}
n \\
k
\end{array}\right)\left(\begin{array}{c}
n-k \\
j
\end{array}\right) t^{k+j} \partial_{x_{n-k-j}}+p\left(\begin{array}{c}
n \\
\ell
\end{array}\right) t^{n-\ell} \\
& \hat{\pi}_{\delta, p}(C)=-u \partial_{u}^{2}-\sum_{j=0}^{\ell-1}(\ell-j) y_{j} \partial_{y_{j}} \partial_{u}+\sum_{j=0}^{\ell-2}(2 \ell-j) y_{j+1} \partial_{y_{j}}-\left(\delta-\delta_{\rho}\right) \partial_{u} \\
& \quad-\sum_{j=1}^{\ell}(-1)^{j} B_{j}(\ell+1)\left(\begin{array}{c}
\ell \\
j
\end{array}\right) y_{\ell-j} \partial_{y_{\ell-1}} \partial_{u}^{j}-p(\ell+1) \partial_{y_{\ell-1}} \\
&-\sum_{j=2}^{\ell-1} \sum_{k=0}^{\ell-2}(-1)^{j} \frac{2(\ell-1-k)}{k+1} B_{j}\left(\begin{array}{c}
k+1 \\
j
\end{array}\right) y_{k-j+1} \partial_{y_{k}} \partial_{u}^{j}, \\
&-\sum_{j=0}^{\ell-1} \sum_{k=n-\ell+1}^{n-j}(-1)^{k+j} B_{j}\left(\begin{array}{c}
n \\
k
\end{array}\right)\left(\begin{array}{c}
n-k \\
j
\end{array}\right) y_{n-k-j} \partial_{u}^{k+j}+(-1)^{n-\ell} p\left(\begin{array}{c}
n \\
\ell
\end{array}\right) \partial_{u}^{n-\ell} \\
& \hat{\pi}_{\delta, p}\left(P_{n}\right)=
\end{aligned}
$$

for $n=\ell+1, \ell+2, \ldots, 2 \ell$.

Proof. In what follows we use the notation $\operatorname{ad}_{u(x)}$ instead of $\operatorname{ad}(u(x))$. Using (2.23), we obtain

$$
\begin{aligned}
\operatorname{ad}_{u(x)}\left(P_{n}\right) & =\sum_{j=0}^{\ell-1} x_{j}\left[P_{j}, P_{n}\right]+t\left[H, P_{n}\right]=-n t P_{n-1}, \\
\operatorname{ad}_{u(x)}(H) & =\sum_{j=0}^{\ell-1} x_{j}\left[P_{j}, H\right]+t[H, H]=\sum_{j=0}^{\ell-1} j x_{j} P_{j-1}, \\
\operatorname{ad}_{u(x)}(D) & =\sum_{j=0}^{\ell-1} x_{j}\left[P_{j}, D\right]+t[H, D]=-\sum_{j=0}^{\ell-1} 2(\ell-j) x_{j} P_{j}-2 t H, \\
\operatorname{ad}_{u(x)}(C) & =\sum_{j=0}^{\ell-1} x_{j}\left[P_{j}, C\right]+t[H, C]=-\sum_{j=0}^{\ell-1}(2 \ell-j) x_{j} P_{j+1}-t D .
\end{aligned}
$$

By a similar computation to above, we find

$$
\begin{aligned}
\operatorname{ad}_{u(x)}^{k}\left(P_{n}\right) & =(-1)^{k} k !\left(\begin{array}{l}
n \\
k
\end{array}\right) t^{k} P_{n-k}, \\
\operatorname{ad}_{u(x)}^{k}(H) & =\sum_{j=0}^{\ell-1}(-1)^{k-1} k !\left(\begin{array}{l}
j \\
k
\end{array}\right) t^{k-1} x_{j} P_{j-k}
\end{aligned}
$$

for all $k \in \mathbb{N}$, and

$$
\begin{aligned}
\operatorname{ad}_{u(x)}^{2}(C) & =\sum_{j=0}^{\ell-1}((2 \ell-j)(j+1)+2(\ell-j)) t x_{j} P_{j}+2 t^{2} H \\
\operatorname{ad}_{u(x)}^{k}(D) & =\sum_{j=0}^{\ell-2} 2(-1)^{k} \frac{\ell-1-j}{j+1} k !\left(\begin{array}{c}
j+1 \\
k
\end{array}\right) t^{k-1} x_{j} P_{j-k+1}
\end{aligned}
$$


for $k \geq 2$. By Theorem 1.1 we have

$$
\pi_{\delta, p}(a)=\sum_{i=1}^{\ell+1}\left[\operatorname{ad}_{u(x)}(a)\right]_{i} \partial_{u^{i}}+\left(\lambda_{\delta, p}+\rho\right)(a)
$$

for $a \in \mathfrak{h}$, where $u^{i}=x_{i-1}$ for $i=1,2, \ldots, \ell$ and $u^{\ell+1}=t$. Therefore, using (2.32), we get

$$
\begin{aligned}
& \pi_{\delta, p}(D)=-2 t \partial_{t}-2 \sum_{j=0}^{\ell-1}(\ell-j) x_{j} \partial_{x_{j}}+\delta-\left(1+\frac{1}{2} \ell(\ell+1)\right), \\
& \pi_{\delta, p}\left(P_{\ell}\right)=-\ell t \partial_{x_{\ell-1}}+p .
\end{aligned}
$$

Similarly, from Theorem 1.1 we have

$$
\pi_{\delta, p}(a)=-\sum_{i=1}^{\ell+1}\left[\frac{\operatorname{ad}_{u(x)}}{e^{\operatorname{ad}_{u(x)}-\mathrm{id}_{\mathfrak{n}_{+}}}} a\right]_{i} \partial_{u^{i}}
$$

for $a \in \mathfrak{n}_{+}$, where $u^{i}=x_{i-1}$ for $i=1,2, \ldots, \ell$ and $u^{\ell+1}=t$, which we can rewrite using (2.25) into the form

$$
\pi_{\delta, p}(a)=-\sum_{i=1}^{\ell+1} \sum_{j=0}^{\infty} \frac{B_{j}}{j !}\left[\operatorname{ad}_{u(x)}^{j}(a)\right]_{i} \partial_{u^{i}}
$$

for $a \in \mathfrak{n}_{+}$. Therefore, we get

$$
\begin{aligned}
\pi_{\delta, p}\left(P_{n}\right) & =-\sum_{i=1}^{\ell+1} \sum_{j=0}^{\infty} \frac{B_{j}}{j !}\left[\operatorname{ad}_{u(x)}^{j}\left(P_{n}\right)\right]_{i} \partial_{u^{i}}=-\partial_{x_{n}}-\sum_{j=1}^{\infty}(-1)^{j} B_{j}\left(\begin{array}{c}
n \\
j
\end{array}\right) t^{j} \partial_{x_{n-j}} \\
& =-\partial_{x_{n}}-\sum_{j=1}^{n}(-1)^{j} B_{j}\left(\begin{array}{c}
n \\
j
\end{array}\right) t^{j} \partial_{x_{n-j}}, \\
\pi_{\delta, p}(H) & =-\sum_{i=1}^{\ell+1} \sum_{j=0}^{\infty} \frac{B_{j}}{j !}\left[\operatorname{ad}_{u(x)}^{j}(H)\right]_{i} \partial_{u^{i}}=-\partial_{t}-\sum_{j=1}^{\infty} \sum_{k=0}^{\ell-1}(-1)^{j-1} B_{j}\left(\begin{array}{c}
k \\
j
\end{array}\right) t^{j-1} x_{k} \partial_{x_{k-j}} \\
& =-\partial_{t}-\sum_{j=1}^{\ell-1} \sum_{k=j}^{\ell-1}(-1)^{j-1} B_{j}\left(\begin{array}{c}
k \\
j
\end{array}\right) t^{j-1} x_{k} \partial_{x_{k-j}},
\end{aligned}
$$

where we used (2.33).

Finally, from Theorem 1.1 we have

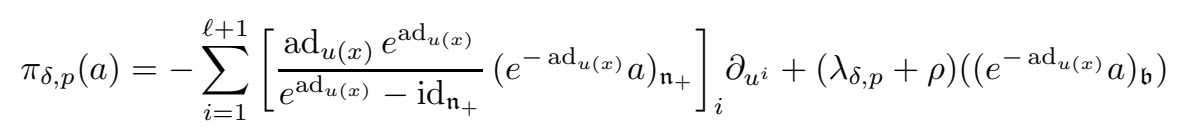

for $a \in \mathfrak{n}_{-}$, where $u^{i}=x_{i-1}$ for $i=1,2, \ldots, \ell$ and $u^{\ell+1}=t$, which we can again rewrite using (2.25) into the form

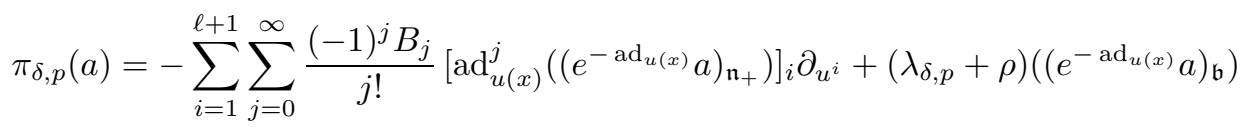

for $a \in \mathfrak{n}_{-}$, where we used the fact $B_{j}(1)=(-1)^{j} B_{j}(0)$ for $j \in \mathbb{N}_{0}$.

Hence, we can write

$$
e^{-\operatorname{ad}_{u(x)}} P_{n}=\sum_{j=0}^{\infty} \frac{(-1)^{j}}{j !} \operatorname{ad}_{u(x)}^{j}\left(P_{n}\right)=\sum_{j=0}^{\infty}\left(\begin{array}{c}
n \\
j
\end{array}\right) t^{j} P_{n-j}=\sum_{j=0}^{n}\left(\begin{array}{c}
n \\
j
\end{array}\right) t^{j} P_{n-j}=\sum_{j=0}^{n}\left(\begin{array}{l}
n \\
j
\end{array}\right) t^{n-j} P_{j},
$$


which gives

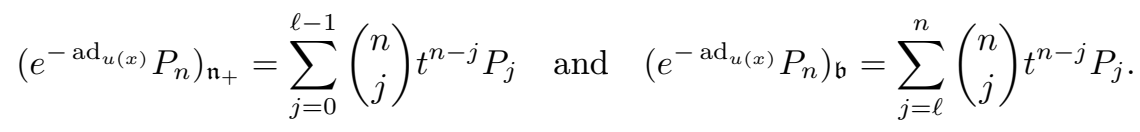

Therefore, we have

$$
\begin{aligned}
\pi_{\delta, p}\left(P_{n}\right) & =-\sum_{i=1}^{\ell+1} \sum_{j=0}^{\infty} \sum_{k=0}^{\ell-1} \frac{(-1)^{j} B_{j}}{j !}\left(\begin{array}{l}
n \\
k
\end{array}\right) t^{n-k}\left[\operatorname{ad}_{u(x)}^{j}\left(P_{k}\right)\right]_{i} \partial_{u^{i}}+\sum_{k=\ell}^{n}\left(\begin{array}{l}
n \\
k
\end{array}\right) t^{n-k}\left(\lambda_{\delta, p}+\rho\right)\left(P_{k}\right) \\
& =-\sum_{j=0}^{\infty} \sum_{k=0}^{\ell-1} B_{j}\left(\begin{array}{c}
n \\
k
\end{array}\right)\left(\begin{array}{c}
k \\
j
\end{array}\right) t^{n-k+j} \partial_{x_{k-j}}+p\left(\begin{array}{c}
n \\
\ell
\end{array}\right) t^{n-\ell} \\
& =-\sum_{j=0}^{\ell-1} \sum_{k=0}^{\ell-1} B_{j}\left(\begin{array}{l}
n \\
k
\end{array}\right)\left(\begin{array}{c}
k \\
j
\end{array}\right) t^{n-k+j} \partial_{x_{k-j}}+p\left(\begin{array}{c}
n \\
\ell
\end{array}\right) t^{n-\ell} \\
& =-\sum_{j=0}^{\ell-1} \sum_{k=n-\ell+1}^{n-j} B_{j}\left(\begin{array}{c}
n \\
k
\end{array}\right)\left(\begin{array}{c}
n-k \\
j
\end{array}\right) t^{k+j} \partial_{x_{n-k-j}}+p\left(\begin{array}{c}
n \\
\ell
\end{array}\right) t^{n-\ell} .
\end{aligned}
$$

From (2.32) and (2.34), we get

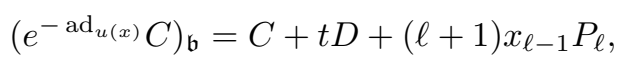

since $\left(\operatorname{ad}_{u(x)}^{k}(C)\right)_{\mathfrak{n}_{+}}=0$ for $k>2$, and

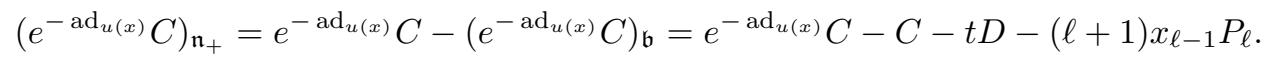

Then we may write

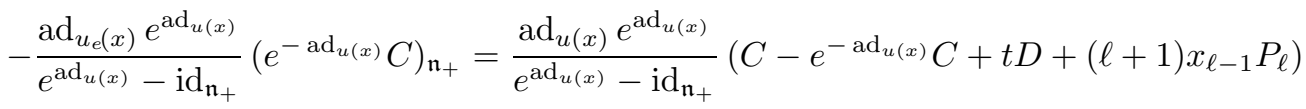

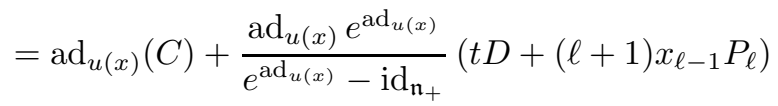

$$
\begin{aligned}
& =\operatorname{ad}_{u(x)}(C)+\sum_{j=0}^{\infty} \frac{(-1)^{j} B_{j}}{j !} \operatorname{ad}_{u(x)}^{j}\left(t D+(\ell+1) x_{\ell-1} P_{\ell}\right),
\end{aligned}
$$

which allows to simplify the right hand side into

$$
\begin{aligned}
-\sum_{j=0}^{\ell-2}(2 \ell-j) x_{j} P_{j+1}-\sum_{j=0}^{\ell-1}(\ell-j) t x_{j} P_{j}-t^{2} H & +\sum_{j=1}^{\infty}(\ell+1) B_{j}\left(\begin{array}{c}
\ell \\
j
\end{array}\right) t^{j} x_{\ell-1} P_{\ell-j} \\
& +\sum_{j=2}^{\infty} \sum_{k=0}^{\ell-2} \frac{2(\ell-1-k)}{k+1} B_{j}\left(\begin{array}{c}
k+1 \\
j
\end{array}\right) t^{j} x_{k} P_{k-j+1} .
\end{aligned}
$$

This, together with

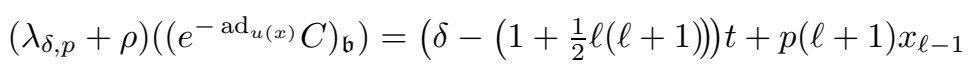

results into the final formula

$$
\begin{aligned}
\pi_{\delta, p}(C)= & -\sum_{j=0}^{\ell-2}(2 \ell-j) x_{j} \partial_{x_{j+1}}-\sum_{j=0}^{\ell-1}(\ell-j) t x_{j} \partial_{x_{j}}-t^{2} \partial_{t}+\sum_{j=1}^{\ell}(\ell+1) B_{j}\left(\begin{array}{l}
\ell \\
j
\end{array}\right) t^{j} x_{\ell-1} \partial_{x_{\ell-j}} \\
& +\sum_{j=2}^{\ell-1} \sum_{k=0}^{\ell-2} \frac{2(\ell-1-k)}{k+1} B_{j}\left(\begin{array}{c}
k+1 \\
j
\end{array}\right) t^{j} x_{k} \partial_{x_{k-j+1}}+\left(\delta-\left(1+\frac{1}{2} \ell(\ell+1)\right)\right) t+p(\ell+1) x_{\ell-1} .
\end{aligned}
$$

The computation of the algebraic Fourier transform of all operators is straightforward. 


\section{Verma modules for conformal Galilei algebras}

Based on the construction of their geometric realization, this section presents a detailed analysis of the composition structure of Verma modules $M_{\ell}(\delta, p)$ for conformal Galilei algebras $\mathfrak{c g a}_{\ell}(1, \mathbb{C})$ with $\ell \in \mathbb{N}$. Namely, we conclude the existence and precise positions of all singular vectors in Verma modules $M_{\ell}(\delta, p)$ for $\ell \in \mathbb{N}$ and $\delta, p \in \mathbb{C}$. Consequently, we shall describe all homomorphisms

$$
\varphi: M_{\ell}(\varepsilon, q) \rightarrow M_{\ell}(\delta, p)
$$

of Verma modules for $\varepsilon, q, \delta, p \in \mathbb{C}$. They are uniquely determined by $\varphi\left(v_{\varepsilon, q}\right) \in M_{\ell}(\delta, p)$, where $v_{\varepsilon, q}$ is the lowest weight vector of $M_{\ell}(\varepsilon, q)$ with lowest weight $\varepsilon \omega_{D}+q \omega_{P_{\ell}}$. By (1.41), we have an isomorphism

$$
\tau \circ \Phi_{\delta, p}: M_{\ell}\left(\delta-\delta_{\rho}, p\right) \stackrel{\sim}{\longrightarrow} \mathbb{C}\left[\mathfrak{n}_{+}^{*}\right]
$$

of $\mathfrak{g}$-modules, where the corresponding action of $\mathfrak{g}$ on $\mathbb{C}\left[\mathfrak{n}_{+}^{*}\right]$ is given through the mapping

$$
\hat{\pi}_{\delta, p}: \mathfrak{g} \rightarrow \mathcal{A}_{\mathfrak{n}_{+}^{*}}^{\mathfrak{g}}
$$

Now, let $v_{\text {sing }} \in \mathbb{C}\left[\mathfrak{n}_{+}^{*}\right]$ be a singular vector with a character $\varepsilon \widetilde{\omega}_{D}+q \widetilde{\omega}_{P_{\ell}}$. Then from Theorem 2.1 we obtain that

$$
\hat{\pi}_{\delta, p}\left(P_{\ell}\right) v_{\text {sing }}=q v_{\text {sing }}, \quad \hat{\pi}_{\delta, p}(D) v_{\text {sing }}=\varepsilon v_{\text {sing }}, \quad \hat{\pi}_{\delta, p}(C) v_{\text {sing }}=0, \quad \hat{\pi}_{\delta, p}\left(P_{n}\right) v_{\text {sing }}=0
$$

for $n=\ell+1, \ell+2, \ldots, 2 \ell$ and some $\varepsilon, q \in \mathbb{C}$. Since the only eigenvalue of the operator $\hat{\pi}_{\delta, p}\left(P_{\ell}\right)=$ $\ell y_{\ell-1} \partial_{u}+p$ on $\mathbb{C}\left[\mathfrak{n}_{+}^{*}\right]$ is $p$, we obtain immediately that the singular vector $v_{\text {sing }} \in \mathbb{C}\left[\mathfrak{n}_{+}^{*}\right]$ does not depend on the variable $u$.

Let us define a Lie subalgebra $\mathfrak{n}_{+, 0}$ of $\mathfrak{n}_{+}$and its complement $\mathfrak{n}_{+, 1}$ in $\mathfrak{n}_{+}$by

$$
\mathfrak{n}_{+, 0}=\left\langle P_{0}, P_{1}, \ldots, P_{\ell-1}\right\rangle \text { and } \mathfrak{n}_{+, 1}=\langle H\rangle .
$$

Then the decomposition

$$
\mathfrak{n}_{+}=\mathfrak{n}_{+, 0} \oplus \mathfrak{n}_{+, 1}
$$

of the vector space $\mathfrak{n}_{+}$gives rise to a canonical isomorphism

$$
\mathbb{C}\left[\mathfrak{n}_{+}^{*}\right] \simeq \mathbb{C}\left[\mathfrak{n}_{+, 0}^{*}\right] \otimes_{\mathbb{C}} \mathbb{C}\left[\mathfrak{n}_{+, 1}^{*}\right]
$$

of commutative $\mathbb{C}$-algebras. Because $v_{\text {sing }} \in \mathbb{C}\left[\mathfrak{n}_{+}^{*}\right]$ does not depend on the variable $u$, we have $v_{\text {sing }} \in \mathbb{C}\left[\mathfrak{n}_{+, 0}^{*}\right]$ and the system of equations (3.4) reduces to

$$
\hat{\pi}_{\delta, p}^{\mathrm{res}}\left(P_{\ell}\right) v_{\mathrm{sing}}=q v_{\mathrm{sing}}, \quad \hat{\pi}_{\delta, p}^{\mathrm{res}}(D) v_{\mathrm{sing}}=\varepsilon v_{\mathrm{sing}}, \quad \hat{\pi}_{\delta, p}^{\mathrm{res}}(C) v_{\text {sing }}=0
$$

for some $\varepsilon, q \in \mathbb{C}$, where

$$
\begin{array}{r}
\hat{\pi}_{\delta, p}^{\mathrm{res}}\left(P_{\ell}\right)=p, \quad \hat{\pi}_{\delta, p}^{\mathrm{res}}(D)=\sum_{j=0}^{\ell-1} 2(\ell-j) y_{j} \partial_{y_{j}}+\delta-\delta_{\rho} \\
\hat{\pi}_{\delta, p}^{\mathrm{res}}(C)=\sum_{j=0}^{\ell-2}(2 \ell-j) y_{j+1} \partial_{y_{j}}-p(\ell+1) \partial_{y_{\ell-1}}
\end{array}
$$

It is more convenient to consider the following change of linear coordinate functions on $\mathfrak{n}_{+, 0}^{*}$ defined by

$$
z_{n}=\frac{y_{\ell-1-n}}{(\ell+1+n) !} \quad \text { for } \quad n=0,1, \ldots, \ell-1
$$


with the inverse given by

$$
y_{n}=(2 \ell-n) ! z_{\ell-1-n} \quad \text { for } \quad n=0,1, \ldots, \ell-1 .
$$

Let us note that the grading of the Lie algebra $\mathfrak{g}$ gives us a natural grading on the commutative $\mathbb{C}$ algebra $\mathbb{C}\left[\mathfrak{n}_{+, 0}^{*}\right]$ defined by $\operatorname{deg} y_{n}=\ell-n$ for $n=0,1, \ldots, \ell-1$. Then (3.10) implies $\operatorname{deg} z_{n}=n+1$ for $n=0,1, \ldots, \ell-1$.

Lemma 3.1. Let $\delta, p \in \mathbb{C}$. Then as operators in the variables $z_{0}, z_{1}, \ldots, z_{\ell-1}$, we have
1) $\hat{\pi}_{\delta, p}^{\mathrm{res}}\left(P_{\ell}\right)=p$,
2) $\hat{\pi}_{\delta, p}^{\mathrm{res}}(D)=\sum_{n=0}^{\ell-1} 2(n+1) z_{n} \partial_{z_{n}}+\delta-\delta_{\rho}$,
3) $\hat{\pi}_{\delta, p}^{\mathrm{res}}(C)=\sum_{n=0}^{\ell-2} z_{n} \partial_{z_{n+1}}-\frac{p}{\ell !} \partial_{z_{0}}$.

Proof. The proof is a straightforward change of coordinates on $\mathfrak{n}_{+, 0}^{*}$.

Let us denote by $F=\mathbb{C}\left[z_{0}, z_{1}, \ldots\right]$ the $\mathbb{C}$-algebra of polynomials in infinitely many variables $z_{n}$, $n \in \mathbb{N}_{0}$, and by $F_{\ell}=\mathbb{C}\left[z_{0}, z_{1}, \ldots, z_{\ell-1}\right]$ for $\ell \in \mathbb{N}$ its finitely generated $\mathbb{C}$-subalgebra of polynomials in the variables $z_{n}, n=0,1, \ldots, \ell-1$. Furthermore, let us consider a first order linear differential operator

$$
T_{1}^{c}=\sum_{n=0}^{\infty} z_{n} \partial_{z_{n+1}}-c \partial_{z_{0}}
$$

on $F$ for $c \in \mathbb{C}$. Then $F_{\ell}$ for $\ell \in \mathbb{N}$ is clearly an invariant subspace of $T_{1}^{c}$ for all $c \in \mathbb{C}$.

Let $v_{\text {sing }} \in F_{\ell} \simeq \mathbb{C}\left[\mathfrak{n}_{+, 0}^{*}\right]$ be a singular vector in $M_{\ell}\left(\delta-\delta_{\rho}, p\right) \simeq \mathbb{C}\left[\mathfrak{n}_{+}^{*}\right]$ with the character $\varepsilon \widetilde{\omega}_{D}+q \widetilde{\omega}_{P_{\ell}}$. Then by Lemma 3.1 we have

$$
\hat{\pi}_{\delta, p}^{\mathrm{res}}\left(P_{\ell}\right) v_{\text {sing }}=q v_{\text {sing }} \quad \text { and } \quad \hat{\pi}_{\delta, p}^{\mathrm{res}}(D) v_{\text {sing }}=\varepsilon v_{\text {sing }},
$$

which implies that the singular vector $v_{\text {sing }}$ is a graded homogeneous polynomial of homogeneity $h=\frac{1}{2}\left(\varepsilon-\delta+\delta_{\rho}\right)$ with respect to the grading $\operatorname{deg} z_{n}=n+1$ for $n \in \mathbb{N}_{0}$, and that $q=p$. Finally, the equation $\hat{\pi}_{\delta, p}^{\text {res }}(C) v_{\text {sing }}=0$ gives $v_{\text {sing }} \in \operatorname{ker} T_{1}^{c}$ with $c=\frac{p}{\ell !}$.

On the other hand, let $v \in F$ be a graded homogeneous polynomial of homogeneity $h \in \mathbb{N}_{0}$ and let $v \in \operatorname{ker} T_{1}^{c}$ for some $c \in \mathbb{C}$. Obviously, there exists a smallest $\ell_{0} \in \mathbb{N}$ such that $v \in F_{\ell_{0}}$. Then for all $\ell \geq \ell_{0}$, we have $v \in F_{\ell}$ and

$$
\begin{gathered}
\hat{\pi}_{\delta, \ell ! c}^{\mathrm{res}}\left(P_{\ell}\right) v=\ell ! c v \\
\hat{\pi}_{\delta, \ell ! c}^{\mathrm{res}}(D) v=\left(\sum_{n=0}^{\ell-1} 2(n+1) z_{n} \partial_{z_{n}}+\delta-\delta_{\rho}\right) v=\left(2 h+\delta-\delta_{\rho}\right) v, \\
\hat{\pi}_{\delta, \ell ! c}^{\mathrm{res}}(C) v=\left(\sum_{n=0}^{\ell-2} z_{n} \partial_{z_{n+1}}-\frac{\ell ! c}{\ell !} \partial_{z_{0}}\right) v=0,
\end{gathered}
$$

which means that $v$ is a singular vector in $M_{\ell}\left(\delta-\delta_{\rho}, \ell ! c\right) \simeq \mathbb{C}\left[\mathfrak{n}_{+}^{*}\right]$ for $\mathfrak{c g a}_{\ell}(1, \mathbb{C})$ with character $\left(2 h+\delta-\delta_{\rho}\right) \widetilde{\omega}_{D}+\ell ! c \widetilde{\omega}_{P_{\ell}}$.

It follows from our considerations in the last two paragraphs that all singular vectors in the Verma module $M_{\ell}\left(\delta-\delta_{\rho}, p\right)$ for $\mathfrak{c g a}_{\ell}(1, \mathbb{C})$ with $\ell \in \mathbb{N}$, can be obtained as graded homogeneous solutions of $T_{1}^{c}$ for some $c \in \mathbb{C}$ on the vector space $F$. This passage is the key observation with several useful consequences, which we shall use through the rest of the article. 


\subsection{Singular vectors and generating series}

The classification of all singular vectors in the Verma module $M_{\ell}(\delta, p)$ for $\mathfrak{c g a} \mathfrak{a}_{\ell}(1, \mathbb{C})$ with $\ell \in \mathbb{N}$ can be rephrased as a task to determine all solutions of the first order linear differential operator

$$
T_{1}^{c}=\sum_{n=0}^{\infty} z_{n} \partial_{z_{n+1}}-c \partial_{z_{0}}, \quad c \in \mathbb{C},
$$

acting on the vector space $F=\mathbb{C}\left[z_{0}, z_{1}, \ldots\right]$ of polynomials in infinitely many variables $z_{n}, n \in \mathbb{N}_{0}$. The aim of this section is to describe all solutions of $T_{1}^{c}$ on $F$. We remark that the standard notation used in the rest of the article and related to symmetric polynomials is for the convenience of the reader reviewed in Appendix $\mathrm{A}$.

We denote by $F^{j}$ the vector subspace of $F$ of graded homogenous (with respect to the grading $\operatorname{deg} z_{n}=n+1$ for $n \in \mathbb{N}_{0}$ ) polynomials of homogeneity $j \in \mathbb{N}_{0}$, and by $F^{j, k}$ the vector subspace of $F$ of graded homogenous (with respect to the grading $\operatorname{deg} z_{n}=n+1$ for $n \in \mathbb{N}_{0}$ ) polynomials of homogeneity $j \in \mathbb{N}_{0}$ and homogeneous (with respect to the grading $\operatorname{deg} z_{n}=1$ for $n \in \mathbb{N}_{0}$ ) polynomials of degree $k \in \mathbb{N}_{0}$. Then there are the decompositions

$$
F=\bigoplus_{j=0}^{\infty} F^{j} \quad \text { and } \quad F=\bigoplus_{k=0}^{\infty} \bigoplus_{j=k}^{\infty} F^{j, k}
$$

Now, let us introduce a generating (or formal power) series $a_{c}(w) \in F[[w]]$ for $c \in \mathbb{C}$ in a formal variable $w$ by

$$
a_{c}(w)=-c+\sum_{n=0}^{\infty} z_{n} w^{n+1}
$$

Further, for $\alpha \in \mathbb{C}$ we have

$$
\begin{aligned}
T_{1}^{c} a_{c}(\alpha w) & =\left(\sum_{k=0}^{\infty} z_{k} \partial_{z_{k+1}}-c \partial_{z_{0}}\right)\left(-c+\sum_{n=0}^{\infty} z_{n}(\alpha w)^{n+1}\right)=-c(\alpha w)+\sum_{k=0}^{\infty} \sum_{n=0}^{\infty} z_{k} \partial_{z_{k+1}} z_{n}(\alpha w)^{n+1} \\
& =-c(\alpha w)+\sum_{k=0}^{\infty} \sum_{n=0}^{\infty} z_{k} \delta_{n, k+1}(\alpha w)^{n+1}=-c(\alpha w)+\sum_{k=0}^{\infty} z_{k}(\alpha w)^{k+2}=\alpha w a_{c}(\alpha w),
\end{aligned}
$$

which implies

$$
T_{1}^{c}\left(\prod_{k=1}^{r} a_{c}\left(\alpha_{k} w\right)\right)=\left(\sum_{k=1}^{r} \alpha_{k}\right) w \prod_{k=1}^{r} a_{c}\left(\alpha_{k} w\right)
$$

for all $r \in \mathbb{N}$ and $\alpha_{1}, \alpha_{2}, \ldots, \alpha_{r} \in \mathbb{C}$. Therefore, from (3.18) it follows that the coefficient by $w^{n}$ in the expansion of $\prod_{k=1}^{r} a_{c}\left(\alpha_{k} w\right)$ is in $\operatorname{ker} T_{1}^{c}$ provided $\sum_{k=1}^{r} \alpha_{k}=0$.

Our next step is to find the coefficient by $w^{n}$ in the expansion of $\prod_{k=1}^{r} a_{c}\left(\alpha_{k} w\right)$ for $r \in \mathbb{N}$ and $\alpha_{1}, \alpha_{2}, \ldots, \alpha_{r} \in \mathbb{C}$. Since this expansion depends on whether or not $c$ is zero, we have to discuss these two cases separately.

Case $c \neq 0$. In order to determine the coefficient by $w^{n}$, we make use of the following trick. We can formally write

$$
-c+\sum_{i=0}^{n-1} z_{i} w^{i+1}=-c \prod_{i=1}^{n}\left(1+\gamma_{i} w\right)=-c \sum_{i=0}^{n} e_{i}\left(\gamma_{1}, \gamma_{2}, \ldots, \gamma_{n}\right) w^{i},
$$

where

$$
e_{0}\left(\gamma_{1}, \gamma_{2}, \ldots, \gamma_{n}\right)=1, \quad e_{i}\left(\gamma_{1}, \gamma_{2}, \ldots, \gamma_{n}\right)=-\frac{z_{i-1}}{c}
$$


for $i=1,2, \ldots, n$ are the elementary symmetric polynomials. Therefore, we have

$$
\begin{aligned}
\prod_{k=1}^{r} a_{c}\left(\alpha_{k} w\right) \bmod \left(w^{n+1}\right) & =\prod_{k=1}^{r}(-c) \prod_{i=1}^{n}\left(1+\gamma_{i} \alpha_{k} w\right) \bmod \left(w^{n+1}\right) \\
& =(-c)^{r} \prod_{i=1}^{n} \prod_{k=1}^{r}\left(1+\gamma_{i} \alpha_{k} w\right) \bmod \left(w^{n+1}\right)
\end{aligned}
$$

for all $n \in \mathbb{N}_{0}$, where we always regard the empty product equal to 1 . By (A.29), we get

$$
\prod_{i=1}^{n} \prod_{k=1}^{r}\left(1+\gamma_{i} \alpha_{k} w\right)=\sum_{\lambda \in \mathcal{P}} \varepsilon_{\lambda} \frac{p_{\lambda}\left(\alpha_{1}, \ldots, \alpha_{r}\right) p_{\lambda}\left(\gamma_{1}, \ldots, \gamma_{n}\right)}{z_{\lambda}} w^{|\lambda|}
$$

which enables us to rewrite (3.21) into the form

$$
\prod_{k=1}^{r} a_{c}\left(\alpha_{k} w\right) \bmod \left(w^{n+1}\right)=(-c)^{r} \sum_{k=0}^{n} \sum_{\lambda \in \mathcal{P}_{k}} \varepsilon_{\lambda} \frac{p_{\lambda}\left(\alpha_{1}, \ldots, \alpha_{r}\right) p_{\lambda}\left(\gamma_{1}, \ldots, \gamma_{n}\right)}{z_{\lambda}} w^{k} .
$$

Using (3.20) and the Newton's identities (A.20), the polynomial $p_{k}\left(\gamma_{1}, \ldots, \gamma_{n}\right)$ can be written as a graded homogenous (with respect to the grading $\operatorname{deg} z_{n}=n+1$ for $n \in \mathbb{N}_{0}$ ) polynomial of homogeneity $k$ in the variables $z_{0}, z_{1}, \ldots, z_{k-1}$ provided $k \leq n$. Hence, we have

$$
p_{k}\left(\gamma_{1}, \ldots, \gamma_{n}\right)=u_{k}\left(-\frac{z_{0}}{c}, \ldots,-\frac{z_{k-1}}{c}\right)=\frac{t_{k}^{c}(z)}{(-1)^{k} c^{k}}
$$

as follows from (A.22), where the polynomial $t_{k}^{c}(z) \in F^{k}$ and does not depend on $n$. Let us note that the polynomial $t_{k}^{c}(z)$ has a well-defined limit for $c \rightarrow 0$, and we get $t_{k}^{0}(z)=z_{0}^{k}$ for $k \in \mathbb{N}$. Therefore, we may write

$$
\prod_{k=1}^{r} a_{c}\left(\alpha_{k} w\right) \bmod \left(w^{n+1}\right)=(-c)^{r} \sum_{\lambda \in \mathcal{P}} \varepsilon_{\lambda} \frac{p_{\lambda}\left(\alpha_{1}, \ldots, \alpha_{r}\right) t_{\lambda}^{c}(z)}{z_{\lambda}} \frac{w^{|\lambda|}}{(-1)^{|\lambda|} c^{|\lambda|}} \bmod \left(w^{n+1}\right)
$$

for all $n \in \mathbb{N}_{0}$, where $t_{\lambda}^{c}(z)=\prod_{i \in \mathbb{N}} t_{i}^{c}(z)^{m_{i}(\lambda)}$, which gives

$$
\prod_{k=1}^{r} a_{c}\left(\alpha_{k} w\right)=(-c)^{r} \sum_{\lambda \in \mathcal{P}} \varepsilon_{\lambda} \frac{p_{\lambda}\left(\alpha_{1}, \ldots, \alpha_{r}\right) t_{\lambda}^{c}(z)}{z_{\lambda}} \frac{w^{|\lambda|}}{(-1)^{|\lambda|} c^{|\lambda|}} .
$$

We obtain the following power series expansion

$$
\prod_{k=1}^{r} a_{c}\left(\alpha_{k} w\right)=\sum_{n=0}^{\infty} \sum_{\lambda \in \mathcal{P}_{n}} p_{\lambda}\left(\alpha_{1}, \ldots, \alpha_{r}\right) \frac{\varepsilon_{\lambda} t_{\lambda}^{c}(z)}{z_{\lambda}} \frac{w^{n}}{(-1)^{n+r} c^{n+r}}
$$

for all $r \in \mathbb{N}$ and $\alpha_{1}, \alpha_{2}, \ldots, \alpha_{r} \in \mathbb{C}$.

Case $c=0$. We can again formally write

$$
\sum_{i=0}^{n} z_{i} w^{i+1}=z_{0} w \prod_{i=1}^{n}\left(1+\beta_{i} w\right)=z_{0} w \sum_{i=0}^{n} e_{i}\left(\beta_{1}, \beta_{2}, \ldots, \beta_{n}\right) w^{i}
$$

where

$$
e_{i}\left(\beta_{1}, \beta_{2}, \ldots, \beta_{n}\right)=\frac{z_{i}}{z_{0}}
$$


for $i=0,1, \ldots, n$ are the elementary symmetric polynomials. Therefore, we have

$$
\begin{aligned}
\prod_{k=1}^{r} a_{0}\left(\alpha_{k} w\right) \bmod \left(w^{n+2}\right) & =\prod_{k=1}^{r} z_{0} w \prod_{i=1}^{n}\left(1+\beta_{i} \alpha_{k} w\right) \bmod \left(w^{n+2}\right) \\
& =\left(z_{0} w\right)^{r} \prod_{i=1}^{n} \prod_{k=1}^{r}\left(1+\beta_{i} \alpha_{k} w\right) \bmod \left(w^{n+2}\right)
\end{aligned}
$$

for all $n \in \mathbb{N}_{0}$. Now, from (A.29) we get

$$
\prod_{i=1}^{n} \prod_{k=1}^{r}\left(1+\beta_{i} \alpha_{k} w\right)=\sum_{\lambda \in \mathcal{P}} \varepsilon_{\lambda} \frac{p_{\lambda}\left(\alpha_{1}, \ldots, \alpha_{r}\right) p_{\lambda}\left(\beta_{1}, \ldots, \beta_{n}\right)}{z_{\lambda}} w^{|\lambda|},
$$

which enables us to rewrite (3.30) into the form

$$
\prod_{k=1}^{r} a_{0}\left(\alpha_{k} w\right) \bmod \left(w^{n+1+r}\right)=\left(z_{0} w\right)^{r} \sum_{k=0}^{n} \sum_{\lambda \in \mathcal{P}_{k}} \varepsilon_{\lambda} \frac{p_{\lambda}\left(\alpha_{1}, \ldots, \alpha_{r}\right) p_{\lambda}\left(\beta_{1}, \ldots, \beta_{n}\right)}{z_{\lambda}} w^{k} .
$$

Using (3.29) and the Newton's identities (A.20), the polynomial $p_{k}\left(\beta_{1}, \ldots, \beta_{n}\right)$ can be written as a graded homogenous (with respect to the grading $\operatorname{deg} \frac{z_{n}}{z_{0}}=n$ for $n \in \mathbb{N}$ ) polynomial of homogeneity $k$ in the variables $\frac{z_{1}}{z_{0}}, \frac{z_{2}}{z_{0}}, \ldots, \frac{z_{k}}{z_{0}}$ provided $k \leq n$. Hence, we have

$$
p_{k}\left(\beta_{1}, \ldots, \beta_{n}\right)=u_{k}\left(\frac{z_{1}}{z_{0}}, \ldots, \frac{z_{k}}{z_{0}}\right)=\frac{t_{k}(z)}{z_{0}^{k}}
$$

as follows from (A.22), where the polynomial $t_{k}(z) \in F^{2 k, k}$ and does not depend on $n$. Therefore, we may write

$$
\prod_{k=1}^{r} a_{0}\left(\alpha_{k} w\right) \bmod \left(w^{n+1+r}\right)=\left(z_{0} w\right)^{r} \sum_{\lambda \in \mathcal{P}} \varepsilon_{\lambda} \frac{p_{\lambda}\left(\alpha_{1}, \ldots, \alpha_{r}\right) t_{\lambda}(z)}{z_{\lambda}} \frac{w^{|\lambda|}}{z_{0}^{|\lambda|}} \bmod \left(w^{n+1+r}\right)
$$

for all $n \in \mathbb{N}_{0}$, where $t_{\lambda}(z)=\prod_{i \in \mathbb{N}} t_{i}(z)^{m_{i}(\lambda)}$, which gives

$$
\prod_{k=1}^{r} a_{0}\left(\alpha_{k} w\right)=\left(z_{0} w\right)^{r} \sum_{\lambda \in \mathcal{P}} \varepsilon_{\lambda} \frac{p_{\lambda}\left(\alpha_{1}, \ldots, \alpha_{r}\right) t_{\lambda}(z)}{z_{\lambda}} \frac{w^{|\lambda|}}{z_{0}^{|\lambda|}} .
$$

Further, for $\lambda \in \mathcal{P}_{n}$ we have

$$
p_{\lambda}\left(\alpha_{1}, \ldots, \alpha_{r}\right)=\sum_{\mu \in \mathcal{P}_{n}(r)} a_{\lambda, \mu}^{r} p_{\mu}\left(\alpha_{1}, \ldots, \alpha_{r}\right),
$$

where $a_{\lambda, \mu}^{r} \in \mathbb{C}$. Therefore, from (3.35) we get

$$
\begin{aligned}
\prod_{k=1}^{r} a_{0}\left(\alpha_{k} w\right) & =\left(z_{0} w\right)^{r} \sum_{n=0}^{\infty} \sum_{\lambda \in \mathcal{P}_{n}} \varepsilon_{\lambda} \frac{p_{\lambda}\left(\alpha_{1}, \ldots, \alpha_{r}\right) t_{\lambda}(z)}{z_{\lambda}} \frac{w^{n}}{z_{0}^{n}} \\
& =\left(z_{0} w\right)^{r} \sum_{n=0}^{\infty} \sum_{\lambda \in \mathcal{P}_{n}} \sum_{\mu \in \mathcal{P}_{n}(r)} \varepsilon_{\lambda} \frac{a_{\lambda, \mu}^{r} p_{\mu}\left(\alpha_{1}, \ldots, \alpha_{r}\right) t_{\lambda}(z)}{z_{\lambda}} \frac{w^{n}}{z_{0}^{n}} \\
& =\sum_{n=0}^{\infty} \sum_{\mu \in \mathcal{P}_{n}(r)} p_{\mu}\left(\alpha_{1}, \ldots, \alpha_{r}\right)\left(\sum_{\lambda \in \mathcal{P}_{n}} \frac{\varepsilon_{\lambda} a_{\lambda, \mu}^{r}}{z_{\lambda}} t_{\lambda}(z) \frac{z_{0}^{r}}{z_{0}^{n}}\right) w^{n+r}
\end{aligned}
$$

and denoting

$$
s_{\lambda}^{r}(z)=\sum_{\mu \in \mathcal{P}_{n}} \frac{\varepsilon_{\mu} a_{\mu, \lambda}^{r}}{z_{\mu}} t_{\mu}(z) \frac{z_{0}^{r}}{z_{0}^{n}}
$$


for $\lambda \in \mathcal{P}_{n}(r)$, we obtain the following power series expansion

$$
\prod_{k=1}^{r} a_{0}\left(\alpha_{k} w\right)=\sum_{n=0}^{\infty} \sum_{\lambda \in \mathcal{P}_{n}(r)} p_{\lambda}\left(\alpha_{1}, \ldots, \alpha_{r}\right) s_{\lambda}^{r}(z) w^{n+r}
$$

for all $r \in \mathbb{N}$ and $\alpha_{1}, \alpha_{2}, \ldots, \alpha_{r} \in \mathbb{C}$.

\section{Proposition 3.2.}

1) If $c \neq 0$, then the set of polynomials $\left\{t_{\lambda}^{c}(z) ; \lambda \in \mathcal{P}\right\}$ in $F$ is linearly independent.

2) Let us define $s_{\emptyset}^{0}(z)=1$. Then the set of polynomials $\left\{s_{\lambda}^{r}(z) ; r \in \mathbb{N}_{0}, \lambda \in \mathcal{P}(r)\right\}$ in $F$ is linearly independent.

Proof. 1) We have the decomposition $F=\bigoplus_{j=0}^{\infty} F^{j}$. Since $t_{\lambda}^{c}(z) \in F^{|\lambda|}$, which follows from the fact $t_{\lambda}^{c}(z)=\prod_{i \in \mathbb{N}} t_{i}^{c}(z)^{m_{i}(\lambda)}$ and $t_{k}^{c}(z) \in F^{k}$, it is enough to show that the set of polynomials $\left\{t_{\lambda}^{c}(z) ; \lambda \in \mathcal{P}_{n}\right\}$ is linearly independent for all $n \in \mathbb{N}_{0}$. Let us suppose that $\sum_{\lambda \in \mathcal{P}_{n}} a_{\lambda} t_{\lambda}^{c}(z)=0$ for some $a_{\lambda} \in \mathbb{C}$. If $c \neq 0$, then using (3.24), we may write

$$
0=\sum_{\lambda \in \mathcal{P}_{n}} a_{\lambda} t_{\lambda}^{c}(z)=\sum_{\lambda \in \mathcal{P}_{n}}(-1)^{|\lambda|} c^{|\lambda|} a_{\lambda} \frac{t_{\lambda}^{c}(z)}{(-1)^{|\lambda|} c^{|\lambda|}}=\sum_{\lambda \in \mathcal{P}_{n}}(-1)^{|\lambda|} c^{|\lambda|} a_{\lambda} p_{\lambda}\left(\gamma_{1}, \gamma_{2}, \ldots, \gamma_{n}\right)
$$

where

$$
e_{0}\left(\gamma_{1}, \gamma_{2}, \ldots, \gamma_{n}\right)=1, \quad e_{i}\left(\gamma_{1}, \gamma_{2}, \ldots, \gamma_{n}\right)=-\frac{z_{i-1}}{c}
$$

for $i=1,2, \ldots, n$. But the set of polynomials $\left\{p_{\lambda}\left(\gamma_{1}, \gamma_{2}, \ldots, \gamma_{n}\right) ; \lambda \in \mathcal{P}_{n}\right\}$ is linearly independent, hence we have $a_{\lambda}=0$ for all $\lambda \in \mathcal{P}_{n}$, and we are done.

2) We have the decomposition $F=\bigoplus_{k=0}^{\infty} \bigoplus_{j=k}^{\infty} F^{j, k}$. Since we have $s_{\lambda}^{r}(z) \in F^{r+|\lambda|, r}$ for $r \in \mathbb{N}_{0}$ and $\lambda \in \mathcal{P}(r)$, which follows by (3.37) and from the fact $t_{\lambda}(z)=\prod_{i \in \mathbb{N}} t_{i}(z)^{m_{i}(\lambda)}$ and $t_{k}(z) \in F^{2 k, k}$, it is enough to show that the set of polynomials $\left\{s_{\lambda}^{r}(z) ; \lambda \in \mathcal{P}_{n}(r)\right\}$ is linearly independent for all $r, n \in \mathbb{N}_{0}$. As $a_{\mu, \lambda}^{r}=\delta_{\mu, \lambda}$ for $\mu, \lambda \in \mathcal{P}_{n}(r)$, we obtain

$$
s_{\lambda}^{r}(z)=\frac{\varepsilon_{\lambda}}{z_{\lambda}} t_{\lambda}(z) \frac{z_{0}^{r}}{z_{0}^{n}}+\sum_{\mu \in \mathcal{P}_{n} \backslash \mathcal{P}_{n}(r)} \frac{\varepsilon_{\mu} a_{\mu, \lambda}^{r}}{z_{\mu}} t_{\mu}(z) \frac{z_{0}^{r}}{z_{0}^{n}}
$$

for $\lambda \in \mathcal{P}_{n}(r)$. But the set $\left\{t_{\mu}(z) ; \mu \in \mathcal{P}_{n}\right\}$ is linearly independent by a similar argument as in the first part, therefore the set $\left\{s_{\lambda}^{r}(z) ; \lambda \in \mathcal{P}_{n}(r)\right\}$ is also linearly independent, and we are done.

Now, if $\sum_{k=1}^{r} \alpha_{k}=0$, then the power series expansions (3.27) and (3.38) reduce into

$$
\prod_{k=1}^{r} a_{c}\left(\alpha_{k} w\right)= \begin{cases}\sum_{n=0}^{\infty} \sum_{\substack{\lambda \in \mathcal{P}_{n} \\ m_{1}(\lambda)=0}} p_{\lambda}\left(\alpha_{1}, \ldots, \alpha_{r}\right) \frac{\varepsilon_{\lambda} t_{\lambda}^{c}(z)}{z_{\lambda}} \frac{w^{n}}{(-1)^{n+r} c^{n+r}} & \text { for } c \neq 0 \\ \sum_{n=0}^{\infty} \sum_{\substack{\lambda \in \mathcal{P}_{n}(r) \\ m_{1}(\lambda)=0}} p_{\lambda}\left(\alpha_{1}, \ldots, \alpha_{r}\right) s_{\lambda}^{r}(z) w^{n+r} & \text { for } c=0\end{cases}
$$

and as a consequence of (3.18) we obtain that

$$
\sum_{\substack{\lambda \in \mathcal{P}_{n} \\ m_{1}(\lambda)=0}} p_{\lambda}\left(\alpha_{1}, \ldots, \alpha_{r}\right) \frac{\varepsilon_{\lambda} t_{\lambda}^{c}(z)}{z_{\lambda}} \in \operatorname{ker} T_{1}^{c \neq 0} \text { and } \sum_{\substack{\lambda \in \mathcal{P}_{n}(r) \\ m_{1}(\lambda)=0}} p_{\lambda}\left(\alpha_{1}, \ldots, \alpha_{r}\right) s_{\lambda}^{r}(z) \in \operatorname{ker} T_{1}^{c=0}
$$

for all $n \in \mathbb{N}_{0}, r \in \mathbb{N}$ and $\alpha_{1}, \alpha_{2}, \ldots, \alpha_{r} \in \mathbb{C}$ satisfying $\sum_{k=1}^{r} \alpha_{k}=0$. In fact, we shall prove that $s_{\lambda}^{r}(z) \in \operatorname{ker} T_{1}^{0}$ for all $r \in \mathbb{N}$ and $\lambda \in \mathcal{P}(r)$ satisfying $m_{1}(\lambda)=0$, and that $t_{\lambda}^{c}(z) \in \operatorname{ker} T_{1}^{c}$ for all $\lambda \in \mathcal{P}$ satisfying $m_{1}(\lambda)=0$ provided $c \neq 0$. 
Proposition 3.3. The following system of equations

$$
\begin{gathered}
p_{1}\left(\alpha_{1}, \alpha_{2}, \ldots, \alpha_{r}\right)=a_{1}, \\
p_{2}\left(\alpha_{1}, \alpha_{2}, \ldots, \alpha_{r}\right)=a_{2}, \\
\vdots \\
p_{r}\left(\alpha_{1}, \alpha_{2}, \ldots, \alpha_{r}\right)=a_{r}
\end{gathered}
$$

has a unique solution up to a permutation of $\alpha_{1}, \alpha_{2}, \ldots, \alpha_{r}$ for any $a_{1}, a_{2}, \ldots, a_{r} \in \mathbb{C}$.

Proof. We see that the system of equations (3.41) for $a_{1}, a_{2}, \ldots, a_{r} \in \mathbb{C}$ is equivalent to the system of equations

$$
\begin{gathered}
e_{1}\left(\alpha_{1}, \alpha_{2}, \ldots, \alpha_{r}\right)=b_{1}, \\
e_{2}\left(\alpha_{1}, \alpha_{2}, \ldots, \alpha_{r}\right)=b_{2}, \\
\vdots \\
e_{r}\left(\alpha_{1}, \alpha_{2}, \ldots, \alpha_{r}\right)=b_{r}
\end{gathered}
$$

for $b_{1}, b_{2}, \ldots, b_{r} \in \mathbb{C}$, where $a_{1}, a_{2}, \ldots, a_{r}$ and $b_{1}, b_{2}, \ldots, b_{r}$ are related through the formulas (A.20) and (A.19).

Let us consider a polynomial $p(x)=\sum_{i=0}^{r}(-1)^{i} b_{i} x^{r-i}$ with $b_{0}=1$. Let $\alpha_{1}, \alpha_{2}, \ldots, \alpha_{r} \in \mathbb{C}$ be the roots of the polynomial $p(x)$, then we have

$$
\sum_{i=0}^{r}(-1)^{i} b_{i} x^{r-i}=\prod_{i=1}^{r}\left(x-\alpha_{i}\right)=\sum_{i=0}^{r}(-1)^{i} e_{i}\left(\alpha_{1}, \alpha_{2}, \ldots, \alpha_{n}\right) x^{r-i}
$$

which proves the claim.

Lemma 3.4. Let us assume that $f_{0}(z), f_{1}(z), \ldots, f_{n}(z) \in F$. If $\sum_{i=0}^{n} a^{i} f_{i}(z) \in \operatorname{ker} T_{1}^{c}$ for all $a \in \mathbb{C}$, then $f_{i}(z) \in \operatorname{ker} T_{1}^{c}$ for $i=0,1, \ldots, n$.

Proof. We introduce $g_{a}(z)=\sum_{i=0}^{n} a^{i} f_{i}(z)$ for $a \in \mathbb{C}$. Then we have

$$
\left(\begin{array}{ccccc}
1 & a_{0} & a_{0}^{2} & \ldots & a_{0}^{n} \\
1 & a_{1} & a_{1}^{2} & \ldots & a_{1}^{n} \\
\vdots & \vdots & \vdots & \ddots & \vdots \\
1 & a_{n} & a_{n}^{2} & \ldots & a_{n}^{n}
\end{array}\right)\left(\begin{array}{c}
f_{0}(z) \\
f_{1}(z) \\
\vdots \\
f_{n}(z)
\end{array}\right)=\left(\begin{array}{c}
g_{a_{0}}(z) \\
g_{a_{1}}(z) \\
\vdots \\
g_{a_{n}}(z)
\end{array}\right)
$$

for $a_{0}, a_{1}, \ldots, a_{n} \in \mathbb{C}$. If $a_{i} \neq a_{j}$ for $i \neq j$, then the Vandermonde matrix is invertible. Therefore, we can express $f_{i}(z)$ as a $\mathbb{C}$-linear combination of $g_{a_{0}}(z), g_{a_{1}}(z), \ldots, g_{a_{n}}(z)$ for $i=0,1, \ldots, n$. Because $g_{a_{i}}(z) \in \operatorname{ker} T_{1}^{c}$ for $i=0,1, \ldots, n$, we obtain that $f_{i}(z) \in \operatorname{ker} T_{1}^{c}$ for $i=0,1, \ldots, n$.

Let us denote $a^{\lambda}=\prod_{i=1}^{r} a_{i}^{m_{i}(\lambda)}$ for $a_{1}, a_{2}, \ldots, a_{r} \in \mathbb{C}$ and $\lambda \in \mathcal{P}(r)$. Now, if $c=0$, then by Proposition 3.3 and (3.40) we obtain that

$$
\sum_{\substack{\lambda \in \mathcal{P}_{n}(r) \\ m_{1}(\lambda)=0}} a^{\lambda} s_{\lambda}^{r}(z) \in \operatorname{ker} T_{1}^{0}
$$

for all $a_{1}, a_{2}, \ldots, a_{r} \in \mathbb{C}$. Since we have

$$
\sum_{\substack{\lambda \in \mathcal{P}_{n}(r) \\ m_{1}(\lambda)=0}} a^{\lambda} s_{\lambda}^{r}(z)=\sum_{k_{2}=0}^{n} a_{2}^{k_{2}}\left(\sum_{k_{3}=0}^{n} a_{3}^{k_{3}}\left(\ldots\left(\sum_{k_{r}=0}^{n} a_{r}^{k_{r}} f_{0, k_{2}, \ldots, k_{r}}^{r}(z)\right) \ldots\right)\right) \in \operatorname{ker} T_{1}^{0}
$$


where $f_{k_{1}, k_{2}, \ldots, k_{r}}^{r}(z)=s_{\lambda}^{r}(z)$ for a partition $\lambda \in \mathcal{P}_{n}(r)$ satisfying $m_{i}(\lambda)=k_{i}$ for $i=1,2, \ldots, r$, and $f_{k_{1}, k_{2}, \ldots, k_{r}}^{r}(z)=0$ otherwise, the multiple application of Lemma 3.4 gives $s_{\lambda}^{r}(z) \in \operatorname{ker} T_{1}^{0}$ for all $\lambda \in \mathcal{P}_{n}(r)$ satisfying $m_{1}(\lambda)=0$. Further, if $c \neq 0$, then by Proposition 3.3 and (3.40) we obtain that

$$
\sum_{\substack{\lambda \in \mathcal{P}_{n} \\ m_{1}(\lambda)=0}} a^{\lambda} \frac{\varepsilon_{\lambda} t_{\lambda}^{c}(z)}{z_{\lambda}} \in \operatorname{ker} T_{1}^{c}
$$

for all $a_{1}, a_{2}, \ldots, a_{r} \in \mathbb{C}$ provided $r \geq n$. By the same argument as for $c=0$, we get $t_{\lambda}^{c}(z) \in \operatorname{ker} T_{1}^{c}$ for all $\lambda \in \mathcal{P}_{n}$ satisfying $m_{1}(\lambda)=0$.

The last step is to show that we have found all linearly independent solutions of $T_{1}^{c}$ on $F$. To prove this fact, let us consider three first order linear differential operators

$$
\begin{aligned}
T_{1}^{c} & =\sum_{n=0}^{\infty} z_{n} \partial_{z_{n+1}}-c \partial_{z_{0}} \\
T_{0}^{c} & =\sum_{n=0}^{\infty}(n+1) z_{n} \partial_{z_{n}}+\frac{1}{2} c \bar{c} \\
T_{-1}^{c} & =\sum_{n=0}^{\infty}(n+1)(n+2) z_{n+1} \partial_{z_{n}}-\bar{c} z_{0}
\end{aligned}
$$

regarded as endomorphisms of the vector space $F$.

Lemma 3.5. We have the following nontrivial commutation relations

$$
\left[T_{0}^{c}, T_{1}^{c}\right]=-T_{1}^{c}, \quad\left[T_{1}^{c}, T_{-1}^{c}\right]=2 T_{0}^{c}, \quad\left[T_{0}^{c}, T_{-1}^{c}\right]=T_{-1}^{c} .
$$

Proof. A straightforward computation gives

$$
\begin{aligned}
{\left[T_{1}^{c}, T_{-1}^{c}\right] } & =\sum_{n, k \in \mathbb{N}_{0}}(k+1)(k+2)\left[z_{n} \partial_{z_{n+1}}, z_{k+1} \partial_{z_{k}}\right]+c \bar{c} \\
& =\sum_{n, k \in \mathbb{N}_{0}}(k+1)(k+2)\left(\left[z_{n} \partial_{z_{n+1}}, z_{k+1}\right] \partial_{z_{k}}+z_{k+1}\left[z_{n} \partial_{z_{n+1}}, \partial_{z_{k}}\right]\right)+c \bar{c} \\
& =\sum_{n, k \in \mathbb{N}_{0}}(k+1)(k+2)\left(\delta_{n, k} z_{n} \partial_{z_{k}}-\delta_{n, k} z_{k+1} \partial_{z_{n+1}}\right)+c \bar{c} \\
& =\sum_{n \in \mathbb{N}_{0}}((n+1)(n+2)-n(n+1)) z_{n} \partial_{z_{n}}+c \bar{c}=\sum_{n \in \mathbb{N}_{0}} 2(n+1) z_{n} \partial_{z_{n}}+c \bar{c}=2 T_{0}^{c} .
\end{aligned}
$$

Similarly, we have

$$
\begin{aligned}
{\left[T_{0}^{c}, T_{-1}^{c}\right] } & =\sum_{n, k \in \mathbb{N}_{0}}(k+1)(n+1)(n+2)\left[z_{k} \partial_{z_{k}}, z_{n+1} \partial_{z_{n}}\right]-\bar{c} z_{0} \\
& =\sum_{n, k \in \mathbb{N}_{0}}(k+1)(n+1)(n+2)\left(\left[z_{k} \partial_{z_{k}}, z_{n+1}\right] \partial_{z_{n}}+z_{n+1}\left[z_{k} \partial_{z_{k}}, \partial_{z_{n}}\right]\right)-\bar{c} z_{0} \\
& =\sum_{n, k \in \mathbb{N}_{0}}(k+1)(n+1)(n+2)\left(\delta_{n+1, k} z_{k} \partial_{z_{n}}-\delta_{n, k} z_{n+1} \partial_{z_{k}}\right)-\bar{c} z_{0} \\
& =\sum_{n \in N_{0}}(n+1)(n+2)((n+2)-(n+1)) z_{n+1} \partial_{z_{n}}-\bar{c} z_{0} \\
& =\sum_{n \in \mathbb{N}_{0}}(n+1)(n+2) z_{n} \partial_{z_{n+1}}-\bar{c} z_{0}=T_{-1}^{c}
\end{aligned}
$$


and

$$
\begin{aligned}
{\left[T_{0}^{c}, T_{1}^{c}\right] } & =\sum_{n, k \in \mathbb{N}_{0}}(k+1)\left[z_{k} \partial_{z_{k}}, z_{n} \partial_{z_{n+1}}\right]+c \partial_{z_{0}} \\
& =\sum_{n, k \in \mathbb{N}_{0}}(k+1)\left(\left[z_{k} \partial_{z_{k}}, z_{n}\right] \partial_{z_{n+1}}+z_{n}\left[z_{k} \partial_{z_{k}}, \partial_{z_{n+1}}\right]\right)+c \partial_{z_{0}} \\
& =\sum_{n, k \in \mathbb{N}_{0}}(k+1)\left(\delta_{n, k} z_{k} \partial_{z_{n+1}}-\delta_{n+1, k} z_{n} \partial_{z_{k}}\right)+c \partial_{z_{0}} \\
& =\sum_{n \in N_{0}}((n+1)-(n+2)) z_{n} \partial_{z_{n+1}}+c \partial_{z_{0}}=-\sum_{n \in \mathbb{N}_{0}} z_{n} \partial_{z_{n+1}}+c \partial_{z_{0}}=-T_{1}^{c} .
\end{aligned}
$$

This finishes the proof.

Now, from Lemma 3.5 it follows that the Lie algebra generated by the linear differential operators $T_{-1}^{c}, T_{0}^{c}$ and $T_{1}^{c}$ is isomorphic to the Lie algebra $\mathfrak{s l}(2, \mathbb{C})$. Let us denote by

$$
e=\left(\begin{array}{ll}
0 & 1 \\
0 & 0
\end{array}\right), \quad h=\left(\begin{array}{cc}
1 & 0 \\
0 & -1
\end{array}\right), \quad f=\left(\begin{array}{ll}
0 & 0 \\
1 & 0
\end{array}\right)
$$

the standard basis of $\mathfrak{s l}(2, \mathbb{C})$. Then the subspace $\mathfrak{h}=\mathbb{C} h$ is a Cartan subalgebra and the subspace $\mathfrak{b}=\mathbb{C} h \oplus \mathbb{C} e$ is a Borel subalgebra of $\mathfrak{s l}(2, \mathbb{C})$. Let us define $\alpha \in \mathfrak{h}^{*}$ by $\alpha(h)=2$ and $\omega \in \mathfrak{h}^{*}$ by $\omega(h)=1$. The representation $\sigma_{c}: \mathfrak{s l}(2, \mathbb{C}) \rightarrow \mathfrak{g l}(F)$ of $\mathfrak{s l}(2, \mathbb{C})$ is given by

$$
\sigma_{c}(e)=T_{1}^{c}, \quad \sigma_{c}(h)=-2 T_{0}^{c}, \quad \sigma_{c}(f)=-T_{-1}^{c} .
$$

Hence, we have got an infinite-dimensional representation of $\mathfrak{s l}(2, \mathbb{C})$ on the vector space $F$. Moreover, if we introduce an inner product on $F$ by

$$
\left\langle g_{1}, g_{2}\right\rangle=\left.g_{1}^{*}\left(\tilde{\partial}_{z}\right) g_{2}(z)\right|_{z=0},
$$

where $z=\left(z_{0}, z_{1}, \ldots, z_{n}, \ldots\right), \tilde{\partial}_{z}=\left(\partial_{z_{0}}, \frac{1}{2} \partial_{z_{1}}, \ldots, \frac{1}{n !(n+1) !} \partial_{z_{n}}, \ldots\right)$ and $g_{1}^{*}(z)=\overline{g_{1}(\bar{z})}$, then we have

$$
\left\langle T_{k}^{c} g_{1}, g_{2}\right\rangle=\left\langle g_{1}, T_{-k}^{c} g_{2}\right\rangle
$$

for all $g_{1}, g_{2} \in F$ and $k=-1,0,1$. The existence of a contravariant inner product implies that the representations $\sigma_{c}: \mathfrak{s l}(2, \mathbb{C}) \rightarrow \mathfrak{g l}(F)$ of $\mathfrak{s l}(2, \mathbb{C})$ are completely reducible for all $c \in \mathbb{C}$.

Furthermore, for $\lambda \in \mathbb{C}$ we denote by $M_{\mathfrak{b}}(\lambda \omega)$ the Verma $\mathfrak{s l}(2, \mathbb{C})$-module with highest weight $\lambda \omega \in \mathfrak{h}^{*}$, and by $L_{\mathfrak{b}}(\lambda \omega)$ its quotient by the maximal $\mathfrak{s l}(2, \mathbb{C})$-submodule. As a consequence, we obtain that the $\mathfrak{s l}(2, \mathbb{C})$-submodule of $F$ generated by a polynomial $g \in F$ satisfying $\sigma_{c}(h) g=\lambda g$ with $\lambda \in \mathbb{C}$ and $\sigma_{c}(e) g=0$ is isomorphic to $L_{\mathfrak{b}}(\lambda \omega)$.

Case $c \neq 0$. Applying $\sigma_{c}(h)$ to $t_{\lambda}^{c}(z)$ for $\lambda \in \mathcal{P}$, we obtain

$$
\sigma_{c}(h) t_{\lambda}^{c}(z)=-\left(2|\lambda|+|c|^{2}\right) t_{\lambda}^{c}(z)
$$

since $t_{\lambda}^{c}(z)=\prod_{i \in \mathbb{N}} t_{i}^{c}(z)^{m_{i}(\lambda)}$ and $t_{k}^{c}(z) \in F^{k}$. Moreover, if $\lambda \in \mathcal{P}$ satisfies $m_{1}(\lambda)=0$, then we also have

$$
\sigma_{c}(e) t_{\lambda}^{c}(z)=0 .
$$

Therefore, the polynomial $t_{\lambda}^{c}(z)$ is the highest weight vector of highest weight $-\left(2|\lambda|+|c|^{2}\right)$ for all $\lambda \in \mathcal{P}$ satisfying $m_{1}(\lambda)=0$.

Let us denote by $m(j)$ for $j \in \mathbb{N}_{0}$ the dimension of the linear span of $\left\{t_{\lambda}^{c}(z) ; \lambda \in \mathcal{P}, m_{1}(\lambda)=\right.$ $0,|\lambda|=j\}$. Since $t_{\lambda}^{c}(z)$ for $\lambda \in \mathcal{P}$ are linearly independent as follows from Proposition 3.2, we obtain that

$$
m(j)=\#\left\{\lambda \in \mathcal{P} ; m_{1}(\lambda)=0,|\lambda|=j\right\}
$$


for $j \in \mathbb{N}_{0}$. Hence, we have

$$
m(j)=\#\left\{\left(m_{2}, m_{3}, \ldots, m_{j}\right) \in \mathbb{N}_{0}^{j-1} ; \sum_{i=2}^{j} i m_{i}=j\right\}
$$

for $j \in \mathbb{N}_{0}$.

Let $M$ the $\mathfrak{s l}(2, \mathbb{C})$-submodule of $F$ generated by $\left\{t_{\lambda}^{c}(z) ; \lambda \in \mathcal{P}, m_{1}(\lambda)=0\right\}$. We take an orthogonal basis of the linear span of $\left\{t_{\lambda}^{c}(z) ; \lambda \in \mathcal{P}, m_{1}(\lambda)=0\right\}$ consisting of weight vectors, so that each basis vector generates an $\mathfrak{s l}(2, \mathbb{C})$-submodule isomorphic to $L_{\mathfrak{b}}(\lambda \omega)$ for some $\lambda \in \mathbb{C}$. Moreover, these $\mathfrak{s l}(2, \mathbb{C})$-submodules are mutually orthogonal for the inner product (3.50), hence we get

$$
F \supset M \simeq M_{\mathfrak{b}}\left(-|c|^{2} \omega\right) \oplus \bigoplus_{j=2}^{\infty} \bigoplus_{\substack{r_{2}, r_{3}, \cdots \in \mathbb{N}_{0} \\ 2 r_{2}+3 r_{3}+\cdots=j}} M_{b}\left(-\left(2 j+|c|^{2}\right) \omega\right)
$$

where the direct summands are generated by $t_{\emptyset}^{c}(z)$ and $\left\{t_{\lambda}^{c}(z) ; \lambda \in \mathcal{P}, m_{1}(\lambda)=0,|\lambda|=j\right\}$ for $j \geq 2$.

Case $c=0$. Applying $\sigma_{0}(h)$ to $s_{\lambda}^{r}(z)$ for $r \in \mathbb{N}_{0}$ and $\lambda \in \mathcal{P}(r)$, we obtain from (3.37) that

$$
\sigma_{0}(h) s_{\lambda}^{r}(z)=-2(r+|\lambda|) s_{\lambda}^{r}(z),
$$

since $t_{\lambda}(z)=\prod_{i \in \mathbb{N}} t_{i}(z)^{m_{i}(\lambda)}$ and $t_{k}(z) \in F^{2 k, k}$. Moreover, if $\lambda \in \mathcal{P}(r)$ satisfies $m_{1}(\lambda)=0$, then we also have

$$
\sigma_{0}(e) s_{\lambda}^{r}(z)=0 .
$$

Therefore, the polynomial $s_{\lambda}^{r}(z)$ is the highest weight vector of highest weight $-2(r+|\lambda|)$ for all $r \in \mathbb{N}_{0}$ and $\lambda \in \mathcal{P}(r)$ satisfying $m_{1}(\lambda)=0$.

Let us denote by $m(j)$ for $j \in \mathbb{N}_{0}$ the dimension of the linear span of $\left\{s_{\lambda}^{r}(z) ; r \in \mathbb{N}_{0}, \lambda \in\right.$ $\left.\mathcal{P}(r), m_{1}(\lambda)=0, r+|\lambda|=j\right\}$. Since $s_{\lambda}^{r}(z)$ for $r \in \mathbb{N}_{0}, \lambda \in \mathcal{P}(r)$ are linearly independent as follows from Proposition 3.2, we obtain that

$$
m(j)=\#\left\{(r, \lambda) ; r \in \mathbb{N}_{0}, \lambda \in \mathcal{P}(r), m_{1}(\lambda)=0, r+|\lambda|=j\right\}
$$

for $j \in \mathbb{N}_{0}$. Further, for $j, r \in \mathbb{N}_{0}$ let us denote

$$
m(j, r)=\#\left\{\lambda \in \mathcal{P}(r) ; m_{1}(\lambda)=0, r+|\lambda|=j\right\} .
$$

Then we have $m(0)=\#\{(0, \emptyset)\}=1$ and $m(1)=\#\{(1, \emptyset)\}=1$. If $j \geq 2$, then $m(j, 0)=0$ and $m(j, 1)=0$, which gives

$$
m(j)=\sum_{r=2}^{j} m(j, r)
$$

For $j \geq r \geq 2$ we may write

$$
\begin{aligned}
m(j, r) & =\#\left\{\left(m_{2}, m_{3}, \ldots, m_{j}\right) \in \mathbb{N}_{0}^{j-1} ; \sum_{i=2}^{r} i m_{i}=j-r, m_{i}=0 \text { for } i>r\right\} \\
& =\#\left\{\left(m_{2}, m_{3}, \ldots, m_{j}\right) \in \mathbb{N}_{0}^{j-1} ; \sum_{i=2}^{r} i m_{i}=j, m_{r} \geq 1, m_{i}=0 \text { for } i>r\right\},
\end{aligned}
$$

which implies

$$
m(j)=\#\left\{\left(m_{2}, m_{3}, \ldots, m_{j}\right) \in \mathbb{N}_{0}^{j-1} ; \sum_{i=2}^{j} i m_{i}=j\right\}
$$

for $j \geq 2$.

Let $M$ be the $\mathfrak{s l}(2, \mathbb{C})$-submodule of $F$ generated by $\left\{s_{\lambda}^{r}(z) ; r \in \mathbb{N}_{0}, \lambda \in \mathcal{P}(r), m_{1}(\lambda)=0\right\}$. Again, we take an orthogonal basis of the linear span of $\left\{s_{\lambda}^{r}(z) ; r \in \mathbb{N}_{0}, \lambda \in \mathcal{P}(r), m_{1}(\lambda)=0\right\}$ 
consisting of weight vectors, so that each basis vector generates an $\mathfrak{s l}(2, \mathbb{C})$-submodule isomorphic to $L_{\mathfrak{b}}(\lambda \omega)$ for some $\lambda \in \mathbb{C}$ and all these $\mathfrak{s l}(2, \mathbb{C})$-submodules are mutually orthogonal. Hence, we obtain

$$
F \supset M \simeq L_{\mathfrak{b}}(0) \oplus M_{\mathfrak{b}}(-2 \omega) \oplus \bigoplus_{j=2}^{\infty} \bigoplus_{\substack{r_{2}, r_{3}, \cdots \in \mathbb{N}_{0} \\ 2 r_{2}+3 r_{3}+\cdots=j}} M_{b}(-2 j \omega)
$$

where the direct summands are generated by $s_{\emptyset}^{0}(z), s_{\emptyset}^{1}(z)$ and $\left\{s_{\lambda}^{r}(z) ; r \in \mathbb{N}_{0}, \lambda \in \mathcal{P}(r), m_{1}(\lambda)=\right.$ $0, r+|\lambda|=j\}$ for $j \geq 2$.

Let us recall that the character of the Verma module $M_{\mathfrak{b}}(\lambda \omega)$ with highest weight $\lambda \omega \in \mathfrak{h}^{*}$ for $\lambda \in \mathbb{C}$ is given by

$$
\operatorname{ch} M_{\mathfrak{b}}(\lambda \omega)=\frac{e^{\lambda \omega}}{1-e^{-\alpha}}=\frac{q^{-\frac{1}{2} \lambda}}{1-q}
$$

where $q=e^{-\alpha}=e^{-2 \omega}$ is a formal variable.

Theorem 3.6. Let $\sigma_{c}: \mathfrak{s l}(2, \mathbb{C}) \rightarrow \mathfrak{g l}(F)$ be the representation of $\mathfrak{s l}(2, \mathbb{C})$ on $F$. Then we have

1) for $c \neq 0$

$$
F \simeq M_{\mathfrak{b}}\left(-|c|^{2} \omega\right) \oplus \bigoplus_{j=2}^{\infty} \bigoplus_{\substack{r_{2}, r_{3}, \cdots \in \mathbb{N}_{0} \\ 2 r_{2}+3 r_{3}+\cdots=j}} M_{\mathfrak{b}}\left(-\left(2 j+|c|^{2}\right) \omega\right),
$$

2) for $c=0$

$$
F \simeq L_{\mathfrak{b}}(0) \oplus M_{\mathfrak{b}}(-2 \omega) \oplus \bigoplus_{j=2}^{\infty} \bigoplus_{\substack{r_{2}, r_{3}, \cdots \in \mathbb{N}_{0} \\ 2 r_{2}+3 r_{3}+\cdots=j}} M_{\mathfrak{b}}(-2 j \omega)
$$

as $\mathfrak{s l}(2, \mathbb{C})$-modules. Recall the notation $L_{\mathfrak{b}}(0) \simeq \mathbb{C}$ for the trivial simple $\mathfrak{s l}(2, \mathbb{C})$-module.

Proof. Let us denote the right hand side of (3.65) and (3.66) by $M$ depending on the value of a parameter $c \in \mathbb{C}$. Since $M \subset F$, as follows from (3.56) and (3.63), it is enough to compare the formal characters of $F$ and $M$. For the $\mathfrak{s l}(2, \mathbb{C})$-module $F$ we have

$$
\begin{aligned}
\operatorname{ch} F & =\sum_{j=0}^{\infty}\left(\operatorname{dim} F^{j}\right) e^{-\left(2 j+|c|^{2}\right) \omega}=q^{\frac{1}{2}|c|^{2}} \sum_{j=0}^{\infty}\left(\operatorname{dim} F^{j}\right) q^{j}=q^{\frac{1}{2}|c|^{2}} \sum_{j=0}^{\infty} \sum_{\substack{r_{0}, r_{1}, \cdots \in \mathbb{N}_{0} \\
r_{0}+2 r_{1}+3 r_{2}+\cdots=j}} q^{j} \\
& =q^{\frac{1}{2}|c|^{2}} \prod_{j=1}^{\infty} \sum_{k=0}^{\infty}\left(q^{j}\right)^{k}=q^{\frac{1}{2}|c|^{2}} \prod_{j=1}^{\infty} \frac{1}{1-q^{j}}=\frac{q^{\frac{1}{2}|c|^{2}}}{\varphi(q)},
\end{aligned}
$$

where $\varphi(q)=\prod_{j=1}^{\infty}\left(1-q^{j}\right)$ and $F^{j}$ is the vector subspace of $F$ of graded homogeneous (with respect to the grading $\operatorname{deg} z_{n}=n+1$ for $n \in \mathbb{N}_{0}$ ) polynomials of homogeneity $j \in \mathbb{N}_{0}$. On the other hand, for the $\mathfrak{s l}(2, \mathbb{C})$-module $M$ we have

$$
\operatorname{ch} M= \begin{cases}\operatorname{ch} M_{\mathfrak{b}}\left(-|c|^{2} \omega\right)+\sum_{j=2}^{\infty} \sum_{\substack{r_{2}, r_{3}, \cdots \in \mathbb{N}_{0} \\ 2 r_{2}+3 r_{3}+\cdots=j}} \operatorname{ch} M_{\mathfrak{b}}\left(-\left(2 j+|c|^{2}\right) \omega\right) & \text { for } c \neq 0, \\ \operatorname{ch} L_{\mathfrak{b}}(0)+\operatorname{ch} M_{\mathfrak{b}}(-2 \omega)+\sum_{j=2}^{\infty} \sum_{\substack{r_{2}, r_{3}, \cdots \in \mathbb{N}_{0} \\ 2 r_{2}+3 r_{3}+\cdots=j}} \operatorname{ch} M_{\mathfrak{b}}(-2 j \omega) & \text { for } c=0 .\end{cases}
$$


Using $\operatorname{ch} L_{\mathfrak{b}}(0)+\operatorname{ch} M_{\mathfrak{b}}(-2 \omega)=\operatorname{ch} M_{\mathfrak{b}}(0)$, we may write

$$
\begin{aligned}
\operatorname{ch} M & =\operatorname{ch} M_{\mathfrak{b}}\left(-|c|^{2} \omega\right)+\sum_{j=2}^{\infty} \sum_{\substack{r_{2}, r_{3}, \cdots \in \mathbb{N}_{0} \\
2 r_{2}+3 r_{3}+\cdots=j}} \operatorname{ch~} M_{\mathfrak{b}}\left(-\left(2 j+|c|^{2}\right) \omega\right) \\
& =\frac{q^{\frac{1}{2}|c|^{2}}}{1-q}+\sum_{j=2}^{\infty} \sum_{\substack{r_{2}, r_{3}, \cdots \in \mathbb{N}_{0} \\
2 r_{2}+3 r_{3}+\cdots=j}} \frac{q^{j+\frac{1}{2}|c|^{2}}}{1-q}=q^{\frac{1}{2}|c|^{2}}\left(\frac{1}{1-q}+\sum_{j=2}^{\infty} \sum_{\substack{r_{2}, r_{3}, \cdots \in \mathbb{N}_{0} \\
2 r_{2}+3 r_{3}+\cdots=j}} \frac{q^{j}}{1-q}\right) \\
& =q^{\frac{1}{2}|c|^{2}}\left(\sum_{j=0}^{\infty} \sum_{\substack{r_{2}, r_{3}, \cdots \in \mathbb{N}_{0} \\
2 r_{2}+3 r_{3}+\cdots=j}} \frac{q^{j}}{1-q}\right)=\frac{q^{\frac{1}{2}|c|^{2}}}{1-q} \prod_{j=2}^{\infty} \sum_{k=0}^{\infty}\left(q^{j}\right)^{k} \\
& =\frac{q^{\frac{1}{2}|c|^{2}}}{1-q} \prod_{j=2}^{\infty} \frac{1}{1-q^{j}}=\frac{q^{\frac{1}{2}|c|^{2}}}{\varphi(q)},
\end{aligned}
$$

which implies the statement.

In fact, we have proved the following first main theorem, which gives an explicit description of the kernel of the linear differential operator $T_{1}^{c}$ on $F$ for all $c \in \mathbb{C}$.

Theorem 3.7. The set

$$
B_{c}= \begin{cases}\left\{t_{\lambda}^{c}(z) ; \lambda \in \mathcal{P}, m_{1}(\lambda)=0\right\} & \text { for } c \neq 0, \\ \left\{s_{\lambda}^{r}(z) ; r \in \mathbb{N}_{0}, \lambda \in \mathcal{P}(r), m_{1}(\lambda)=0\right\} & \text { for } c=0\end{cases}
$$

forms a basis of $\operatorname{ker} T_{1}^{c}$ for all $c \in \mathbb{C}$.

Now, we can formulate our second main theorem, which describes explicitly all singular vectors in the Verma module $M_{\ell}(\delta, p)$ for the conformal Galilei algebra $\mathfrak{c g a}_{\ell}(1, \mathbb{C})$ with $\ell \in \mathbb{N}$.

Theorem 3.8. Let $\delta, p \in \mathbb{C}$ and $p \neq 0$. Then the vector space of singular vectors in the Verma module $M_{\ell}(\delta, p)$ for the conformal Galilei algebra $\mathfrak{c g a}_{\ell}(1, \mathbb{C})$ with $\ell \in \mathbb{N}$ is a linear span of singular vectors

$$
t_{\lambda}^{\frac{p}{\ell ! !}}\left(-\frac{P_{\ell-1}}{(\ell+1) !},-\frac{P_{\ell-2}}{(\ell+2) !}, \ldots,-\frac{P_{0}}{(2 \ell) !}\right) v_{\delta, p}
$$

with character $(\delta+2|\lambda|) \widetilde{\omega}_{D}+p \widetilde{\omega}_{P_{\ell}}$, where $\lambda \in \mathcal{P}(\ell)$ with $m_{1}(\lambda)=0$. Here $v_{\delta, p}$ is the lowest weight vector of $M_{\ell}(\delta, p)$ with lowest weight $\delta \omega_{D}+p \omega_{P_{\ell}}$, and the polynomials $t_{\lambda}^{c}(z) \in F$ are defined by

$$
t_{\lambda}^{c}(z)=\prod_{i \in \mathbb{N}} t_{i}^{c}(z)^{m_{i}(\lambda)}
$$

with

$$
\begin{aligned}
t_{k}^{c}\left(z_{0}, z_{1}, \ldots, z_{k-1}\right) & =(-1)^{k} c^{k} u_{k}\left(-\frac{z_{0}}{c}, \ldots,-\frac{z_{k-1}}{c}\right) \\
& =\sum_{\substack{r_{1}+2 r_{2}+\cdots+k r_{k}=k \\
r_{1}, r_{2}, \ldots, r_{k} \in \mathbb{N}_{0}}} \frac{k\left(r_{1}+\cdots+r_{k}-1\right) !}{r_{1} ! \ldots r_{k} !} \prod_{i=1}^{k}\left(c^{i-1} z_{i-1}\right)^{r_{i}}
\end{aligned}
$$

for $c \in \mathbb{C}$.

Proof. The statement is a straightforward consequence of Theorem 3.7 considerations after Lemma 3.1. the transformation formula (3.10) and the symmetrization map in Theorem 1.4 .

The formulation of an analogous result for $p=0$ is much more delicate as follows from the subsequent example. The explicit form of polynomials $s_{\lambda}^{r}(z)$ in (3.73) implies $s_{\left(2^{3}\right)}^{3}(z), s_{\left(3^{2}\right)}^{3}(z) \in$ $F_{7}$. However, we obtain $4 s_{\left(2^{3}\right)}^{3}(z)-3 s_{\left(3^{2}\right)}^{3}(z) \in F_{5}$, which implies that

$$
s_{\left(2^{3}\right)}^{3}\left(-\frac{P_{\ell-1}}{(\ell+1) !},-\frac{P_{\ell-2}}{(\ell+2) !}, \ldots,-\frac{P_{0}}{(2 \ell) !}\right) v_{\delta, 0}, \quad s_{\left(3^{2}\right)}^{2}\left(-\frac{P_{\ell-1}}{(\ell+1) !},-\frac{P_{\ell-2}}{(\ell+2) !}, \ldots,-\frac{P_{0}}{(2 \ell) !}\right) v_{\delta, 0}
$$


are singular vectors in $M_{\ell}(\delta, 0)$ for $\ell \geq 7$ with a character $(\delta+18) \widetilde{\omega}_{D}$, although

$$
\left(4 s_{\left(2^{3}\right)}^{3}-3 s_{\left(3^{2}\right)}^{3}\right)\left(-\frac{P_{\ell-1}}{(\ell+1) !},-\frac{P_{\ell-2}}{(\ell+2) !}, \ldots,-\frac{P_{0}}{(2 \ell) !}\right) v_{\delta, 0}
$$

is a singular vector in $M_{\ell}(\delta, 0)$ already for $\ell \geq 5$ with character $(\delta+18) \widetilde{\omega}_{D}$. We may at least claim that

$$
s_{\lambda}^{r}\left(-\frac{P_{\ell-1}}{(\ell+1) !},-\frac{P_{\ell-2}}{(\ell+2) !}, \ldots,-\frac{P_{0}}{(2 \ell) !}\right) v_{\delta, 0}
$$

is a singular vector in $M_{\ell}(\delta, p)$ for $\ell \geq \ell_{0}$, where $\ell_{0}$ is the smallest natural number such that $s_{\lambda}^{r}(z) \in F_{\ell_{0}}$, with character $(\delta+2|\lambda|+2 r) \widetilde{\omega}_{D}$ for $r \in \mathbb{N}_{0}, \lambda \in \mathcal{P}(r)$ with $m_{1}(\lambda)=0$ and $v_{\delta, 0}$ the lowest weight vector of $M_{\ell}(\delta, 0)$ with lowest weight $\delta \omega_{D}$.

We give explicit formulas for a few polynomials $s_{\lambda}^{r}(z)$ of small degree with $m_{1}(\lambda)=0$. We have

$$
s_{\emptyset}^{r}(z)=z_{0}^{r}
$$

for all $r \in \mathbb{N}_{0}$, and

$$
\begin{aligned}
& s_{\left(2^{a}\right)}^{2}(z)=\frac{1}{2^{a}} \sum_{\substack{i, j \in \mathbb{N}_{0} \\
i+j=2 a}}(-1)^{i} z_{i} z_{j}, \\
& s_{\left(2^{a}\right)}^{3}(z)=\frac{1}{2^{a}} \sum_{\substack{i, j \in \mathbb{N}_{0} \\
i+j=2 a}}(-1)^{i} z_{0} z_{i} z_{j}=z_{0} s_{\left(2^{a}\right)}^{2}, \\
& s_{\left(3^{a}\right)}^{3}(z)=\frac{1}{3^{a}} \sum_{\substack{i, j, k \in \mathbb{N}_{0} \\
i+j+k=3 a}}\left(-\frac{1}{2}+\frac{\sqrt{3}}{2} \mathrm{i}\right)^{i+2 j} z_{i} z_{j} z_{k}
\end{aligned}
$$

for all $a \in \mathbb{N}_{0}$, which easily follows from (3.38) by a suitable choice of parameters $\alpha_{k} \in \mathbb{C}$ for $k=1,2 \ldots, r$, thus $\alpha_{k}=0$ for $k=1,2, \ldots, r$ in the case $s_{\emptyset}^{r}(z), \alpha_{1}=1, \alpha_{2}=-1$ for $s_{\left(2^{a}\right)}^{2}(z)$, $\alpha_{1}=1, \alpha_{2}=-1, \alpha_{3}=0$ for $s_{\left(2^{a}\right)}^{3}(z)$ and $\alpha_{1}=\omega, \alpha_{2}=\omega^{2}, \alpha_{3}=\omega^{3}$ for $s_{\left(3^{a}\right)}^{3}(z)$, where $\omega=-\frac{1}{2}+\frac{\sqrt{3}}{2} \mathrm{i}$ is the third root of unity.

\subsection{Singular vectors and differential equations of flag type}

The first order linear differential operator $T_{1}^{c}$ of the form (3.15) is a representative of the class of partial differential equations of flag type. A partial differential equation of flag type on a commutative $\mathbb{C}$-algebra $\mathcal{A}=\mathbb{C}\left[x_{1}, x_{2}, \ldots, x_{n}\right]$ is a linear partial differential equation of the form

$$
\left(D_{1}+f_{1} D_{2}+f_{2} D_{3}+\cdots+f_{m-1} D_{m}\right) u=0
$$

for $u \in \mathcal{A}$, where $D_{1}, D_{2}, \ldots, D_{m}$ for $m \in \mathbb{N}$ are commuting locally nilpotent algebraic differential operators on the $\mathbb{C}$-algebra $\mathcal{A}$ and $f_{1}, f_{2}, \ldots, f_{m-1} \in \mathcal{A}$ are polynomials satisfying

$$
D_{k}\left(f_{j}\right)=0 \quad \text { for } k>j .
$$

The results of [14] and [15] gives a parametrization of solution spaces of partial differential equations of flag type (3.74) in the following form. Let $\mathcal{B}$ be a $\mathbb{C}$-subalgebra of $\mathcal{A}$, so that $\mathcal{A}$ is a free $\mathcal{B}$-module generated by a filtered vector subspace $\mathcal{V}=\bigcup_{r=0}^{\infty} \mathcal{V}_{r}, \mathcal{V}_{r} \subset \mathcal{V}_{r+1}$ for $r \in \mathbb{N}_{0}$, of $\mathcal{A}$. Furthermore, let $T_{1}$ be a linear differential operator on $\mathcal{A}$ with a right inverse $T_{1}^{-}$such that

$$
T_{1}(\mathcal{B}) \subset \mathcal{B}, \quad T_{1}^{-}(\mathcal{B}) \subset \mathcal{B}, \quad T_{1}(b v)=T_{1}(b) v, \quad T_{1}^{-}(b v)=T_{1}^{-}(b) v
$$


for all $b \in \mathcal{B}$ and $v \in \mathcal{V}$, and let $T_{2}$ be a linear differential operator on $\mathcal{A}$ such that

$$
T_{2}\left(\mathcal{V}_{r}\right) \subset \mathcal{B} \mathcal{V}_{r-1}, \quad T_{2}(b a)=b T_{2}(a)
$$

for all $a \in \mathcal{A}, b \in \mathcal{B}$ and $r \in \mathbb{N}_{0}$ with the notation $\mathcal{V}_{-1}=\{0\}$. Then the vector subspace of all $u \in \mathcal{A}$ fulfilling $\left(T_{1}+T_{2}\right)(u)=0$ is given by the complex linear span

$$
\left\langle\left\{\sum_{j=0}^{\infty}\left(-T_{1}^{-} T_{2}\right)^{j}(b v) ; v \in \mathcal{V}, b \in \mathcal{B}, T_{1}(b)=0\right\}\right\rangle .
$$

In fact, there are only finitely many contributions in the last summation formula.

In the case of the linear differential operator $T_{1}^{c}, c \neq 0$, which acts on the $\mathbb{C}$-algebra $\mathcal{A}=F_{\ell}=$ $\mathbb{C}\left[z_{0}, z_{1}, \ldots, z_{\ell-1}\right]$ as

$$
-c \partial_{z_{0}}+\sum_{n=0}^{\ell-2} z_{n} \partial_{z_{n+1}}
$$

we take $\mathcal{B}=\mathbb{C}\left[z_{0}\right]$ and $\mathcal{V}=\mathbb{C}\left[z_{1}, z_{2}, \ldots, z_{\ell-1}\right]$ with $\mathcal{V}_{r}=\mathcal{V} \cap \bigoplus_{k=0}^{r} \mathcal{A}_{k}$, where $\mathcal{A}_{k} \subset \mathcal{A}$ is the subspace of graded homogeneous polynomials of homogeneity $k$ with respect to the grading $\operatorname{deg} z_{n}=n+1$ for $n=0,1, \ldots, \ell-1$. Furthermore, if we set

$$
T_{1}=-c \partial_{z_{0}} \quad \text { and } \quad T_{2}=\sum_{n=0}^{\ell-2} z_{n} \partial_{z_{n+1}}
$$

then its right inverse $T_{1}^{-}$is given by

$$
T_{1}^{-}\left(f\left(z_{0}, z_{1}, \ldots, z_{\ell-1}\right)\right)=-\frac{1}{c} \int_{0}^{z_{0}} f\left(z_{0}^{\prime}, z_{1}, \ldots, z_{\ell-1}\right) \mathrm{d} z_{0}^{\prime},
$$

where $\int_{0}^{z_{0}} f\left(z_{0}^{\prime}, z_{1}, \ldots, z_{\ell-1}\right) \mathrm{d} z_{0}^{\prime}$ denotes the definite integration in the variable $z_{0}^{\prime}$. In conclusion, we obtain

$$
\operatorname{ker}\left(-c \partial_{z_{0}}+\sum_{n=0}^{\ell-2} z_{n} \partial_{z_{n}}\right)=\operatorname{ker}\left(T_{1}+T_{2}\right)=\left\{\sum_{j=0}^{\infty}\left(-T_{1}^{-} T_{2}\right)^{j}(v) ; v \in \mathcal{V}\right\}
$$

for $c \neq 0$.

\section{Appendix A Symmetric polynomials and functions}

For the reader's convenience, we summarize several basic facts concerning symmetric polynomials and symmetric functions used throughout the article, see [1] for a detailed information.

Let $S_{n}$ be the symmetric group on the set $\{1,2, \ldots, n\}$ and $\mathbb{C}^{n}$ its defining representation. Then we have the induced representation of $S_{n}$ on the vector space $\mathbb{C}\left[\mathbb{C}^{n}\right]$ of polynomials on $\mathbb{C}^{n}$ defined by

$$
(\sigma \cdot f)(v)=f\left(\sigma^{-1} v\right),
$$

where $\sigma \in S_{n}, f \in \mathbb{C}\left[\mathbb{C}^{n}\right]$ and $v \in \mathbb{C}^{n}$. The subset $\mathbb{C}\left[\mathbb{C}^{n}\right]^{S_{n}}$ of all invariant polynomials is a $\mathbb{C}$-subalgebra of $\mathbb{C}\left[\mathbb{C}^{n}\right]$, and is called the ring of symmetric polynomials.

Let us denote by $x=\left(x_{1}, x_{2}, \ldots, x_{n}\right)$ the canonical linear coordinate functions on $\mathbb{C}^{n}$. Then we have an isomorphism $\mathbb{C}\left[\mathbb{C}^{n}\right] \simeq \mathbb{C}[x]$, and the induced representation of $S_{n}$ on $\mathbb{C}[x]$ is given by

$$
\sigma \cdot f\left(x_{1}, x_{2}, \ldots, x_{n}\right)=f\left(x_{\sigma(1)}, x_{\sigma(2)}, \ldots, x_{\sigma(n)}\right)
$$


for $\sigma \in S_{n}$ and $f\left(x_{1}, x_{2}, \ldots, x_{n}\right) \in \mathbb{C}[x]$. We denote by $\Lambda_{n}(x)$ the ring of $S_{n}$-invariant polynomials in the variables $x_{1}, x_{2}, \ldots, x_{n}$. Moreover, the ring $\Lambda_{n}(x)$ is a graded $\mathbb{C}$-algebra, we have

$$
\Lambda_{n}(x)=\bigoplus_{k=0}^{\infty} \Lambda_{n}^{k}(x)
$$

where $\Lambda_{n}^{k}(x)$ is the vector space of homogeneous symmetric polynomials of degree $k$.

The most important bases of the ring of symmetric polynomials are labeled by partitions of non-negative integers $\mathbb{N}_{0}$. A partition of $n \in \mathbb{N}_{0}$ is a non-increasing sequence $\lambda=\left(\lambda_{1}, \lambda_{2}, \ldots, \lambda_{m}\right)$ of positive integers called parts whose sum is $n$, i.e. $\lambda_{1} \geq \lambda_{2} \geq \cdots \geq \lambda_{m}>0$ and $n=\sum_{i=1}^{m} \lambda_{i}$. The only partition of 0 is the empty partition $\emptyset$. The length $\ell(\lambda)=m$ of $\lambda$ is the length of the sequence and the size $|\lambda|=\sum_{i=1}^{m} \lambda_{i}$ of $\lambda$ is the sum over all elements in the sequence. It is often convenient to define $\lambda_{i}=0$ for $i>\ell(\lambda)$. The set of all partitions of $n$ is denoted by $\mathcal{P}_{n}$, the set of all partitions by $\mathcal{P}$, the set of all partitions of $n$ whose parts are at most $r$ by $\mathcal{P}_{n}(r)$, and the set of all partitions whose parts are at most $r$ by $\mathcal{P}(r)$. Furthermore, for a partition $\lambda$ we define

$$
\varepsilon_{\lambda}=(-1)^{|\lambda|-\ell(\lambda)}, \quad z_{\lambda}=\prod_{i \in \mathbb{N}} i^{m_{i}(\lambda)} m_{i}(\lambda) !, \quad u_{\lambda}=\frac{\ell(\lambda) !}{\prod_{i \in \mathbb{N}} m_{i}(\lambda) !},
$$

where $m_{i}(\lambda)$ indicates the number of times the integer $i$ occurs in the partition $\lambda$, it is called the multiplicity of $i$ in $\lambda$. We shall also use a notation $\left(1^{m_{1}(\lambda)}, 2^{m_{2}(\lambda)}, \ldots, r^{m_{r}(\lambda)}, \ldots\right)$ for a partition $\lambda$.

\section{A.1 Symmetric polynomials}

Here we recall several important classes of symmetric polynomials which are useful in the construction of generating sets of $\Lambda_{n}(x)$.

1) Monomial symmetric polynomials. For a partition $\lambda$ satisfying $\ell(\lambda) \leq n$, we define the symmetric polynomial

$$
m_{\lambda}\left(x_{1}, \ldots, x_{n}\right)=\sum_{\left\{i_{1}, i_{2}, \ldots, i_{\ell(\lambda)}\right\} \subset\{1,2 \ldots, n\}} x_{i_{1}}^{\lambda_{1}} x_{i_{2}}^{\lambda_{2}} \ldots x_{i_{\ell(\lambda)}^{\lambda_{\ell(\lambda)}}}
$$

called monomial symmetric polynomial.

2) Elementary symmetric polynomials. The symmetric polynomials

$$
e_{k}\left(x_{1}, \ldots, x_{n}\right)= \begin{cases}1 & \text { for } k=0 \\ \sum_{1 \leq i_{1}<i_{2}<\cdots<i_{k} \leq n} x_{i_{1}} x_{i_{2}} \ldots x_{i_{k}} & \text { for } k \geq 1\end{cases}
$$

are called elementary symmetric polynomials. For a partition $\lambda$, we define

$$
e_{\lambda}\left(x_{1}, \ldots, x_{n}\right)=\prod_{i=1}^{\ell(\lambda)} e_{\lambda_{i}}\left(x_{1}, \ldots, x_{n}\right) .
$$

3) Power sum symmetric polynomials. The symmetric polynomials

$$
p_{k}\left(x_{1}, \ldots, x_{n}\right)=\sum_{i=1}^{n} x_{i}^{k} \quad \text { for } k \geq 1
$$

are called power sum symmetric polynomials. For a partition $\lambda$, we define

$$
p_{\lambda}\left(x_{1}, \ldots, x_{n}\right)=\prod_{i=1}^{\ell(\lambda)} p_{\lambda_{i}}\left(x_{1}, \ldots, x_{n}\right) .
$$


4) Complete homogeneous symmetric polynomials. The symmetric polynomials

$$
h_{k}\left(x_{1}, \ldots, x_{n}\right)= \begin{cases}1 & \text { for } k=0 \\ \sum_{1 \leq i_{1} \leq i_{2} \leq \cdots \leq i_{k} \leq n} x_{i_{1}} x_{i_{2}} \ldots x_{i_{k}} & \text { for } k \geq 1\end{cases}
$$

are called complete homogeneous symmetric polynomials. For a partition $\lambda$, we define

$$
h_{\lambda}\left(x_{1}, \ldots, x_{n}\right)=\prod_{i=1}^{\ell(\lambda)} h_{\lambda_{i}}\left(x_{1}, \ldots, x_{n}\right) .
$$

Moreover, there is an isomorphism of $\mathbb{C}$-algebras

$$
\Lambda_{n}(x) \simeq \mathbb{C}\left[e_{1}\left(x_{1}, \ldots, x_{n}\right), \ldots, e_{n}\left(x_{1}, \ldots, x_{n}\right)\right] \simeq \mathbb{C}\left[p_{1}\left(x_{1}, \ldots, x_{n}\right), \ldots, p_{n}\left(x_{1}, \ldots, x_{n}\right)\right]
$$

for all $n \in \mathbb{N}$.

\section{A.2 Newton's identities}

We review the Newton's identities, also known as the Newton-Girard formulae, which give relations between power sum and elementary symmetric polynomials.

It is more convenient to work with symmetric polynomials in infinitely than finitely many variables. For any pair $m, n \in \mathbb{N}$ with $m \geq n$, we consider the $\mathbb{C}$-algebra homomorphism

$$
\rho_{m, n}: \Lambda_{m}(x) \rightarrow \Lambda_{n}(x)
$$

defined by

$$
\rho_{m, n}\left(x_{i}\right)= \begin{cases}x_{i} & \text { for } i=1, \ldots, n, \\ 0 & \text { for } i=n+1, \ldots, m\end{cases}
$$

Then the ring of symmetric functions $\Lambda(x)$ in infinitely many variables $x_{n}, n \in \mathbb{N}$, is defined by the inverse limit

$$
\Lambda(x)=\lim _{\longleftarrow n} \Lambda_{n}(x)
$$

together with the projections $\rho_{n}: \Lambda(x) \rightarrow \Lambda_{n}(x)$ given by

$$
\rho_{n}\left(x_{i}\right)= \begin{cases}x_{i} & \text { for } i=1, \ldots, n, \\ 0 & \text { for } i>n .\end{cases}
$$

Let us denote by $e_{n}(x)$ and $p_{n}(x)$ symmetric functions in $\Lambda(x)$ satisfying

$$
\rho_{k}\left(e_{n}(x)\right)=e_{n}\left(x_{1}, x_{2}, \ldots, x_{k}\right) \text { and } \rho_{k}\left(p_{n}(x)\right)=p_{n}\left(x_{1}, x_{2}, \ldots, x_{k}\right)
$$

for all $k \in \mathbb{N}$, respectively. As above, for a partition $\lambda$ we define

$$
e_{\lambda}(x)=\prod_{i=1}^{\ell(\lambda)} e_{\lambda_{i}}(x) \quad \text { and } \quad p_{\lambda}(x)=\prod_{i=1}^{\ell(\lambda)} p_{\lambda_{i}}(x) .
$$

For $n \in \mathbb{N}$, the transformation between elementary and power sum symmetric polynomials is

$$
e_{n}(x)=\sum_{\lambda \in \mathcal{P}_{n}} \varepsilon_{\lambda} \frac{p_{\lambda}(x)}{z_{\lambda}}=\sum_{\substack{r_{1}+2 r_{2}+\cdots+n r_{n}=n \\ r_{1}, r_{2}, \ldots, r_{n} \in \mathbb{N}_{0}}}(-1)^{n} \prod_{i=1}^{n} \frac{\left(-p_{i}(x)\right)^{r_{i}}}{r_{i} ! i^{r_{i}}}
$$


and

$$
p_{n}(x)=n \sum_{\lambda \in \mathcal{P}_{n}} \frac{\varepsilon_{\lambda} u_{\lambda}}{\ell(\lambda)} e_{\lambda}(x)=\sum_{\substack{r_{1}+2 r_{2}+\cdots+n r_{n}=n \\ r_{1}, r_{2}, \ldots, r_{n} \in \mathbb{N}_{0}}}(-1)^{n} \frac{n\left(r_{1}+\cdots+r_{n}-1\right) !}{r_{1} ! \ldots r_{n} !} \prod_{i=1}^{n}\left(-e_{i}(x)\right)^{r_{i}} .
$$

Moreover, if we define polynomials

$$
u_{n}\left(y_{1}, y_{2}, \ldots, y_{n}\right)=\sum_{\substack{r_{1}+2 r_{2}+\cdots+n r_{n}=n \\ r_{1}, r_{2}, \ldots, r_{n} \in \mathbb{N}_{0}}}(-1)^{n} \frac{n\left(r_{1}+\cdots+r_{n}-1\right) !}{r_{1} ! \ldots r_{n} !} \prod_{i=1}^{n}\left(-y_{i}\right)^{r_{i}}
$$

for $n \in \mathbb{N}$, then we may write

$$
p_{n}(x)=u_{n}\left(e_{1}(x), e_{2}(x), \ldots, e_{n}(x)\right)
$$

for all $n \in \mathbb{N}$. Since we have $e_{r}\left(x_{1}, \ldots, x_{n}\right)=0$ for $r>n$, we obtain from fa.20) for $k>n$ the formula

$$
\begin{aligned}
p_{k}\left(x_{1}, \ldots, x_{n}\right) & =k \sum_{\lambda \in \mathcal{P}_{k}(n)} \frac{\varepsilon_{\lambda} u_{\lambda}}{\ell(\lambda)} e_{\lambda}\left(x_{1}, \ldots, x_{n}\right) \\
& =\sum_{\substack{r_{1}+2 r_{2}+\cdots+n r_{n}=k \\
r_{1}, r_{2}, \ldots, r_{n} \in \mathbb{N}_{0}}}(-1)^{k} \frac{k\left(r_{1}+r_{2} \cdots+r_{n}-1\right) !}{r_{1} ! r_{2} ! \ldots r_{n} !} \prod_{i=1}^{n}\left(-e_{i}\left(x_{1}, \ldots, x_{n}\right)\right)^{r_{i}} .
\end{aligned}
$$

The equality (A.19) can be used to produce $p_{k}\left(x_{1}, \ldots, x_{n}\right) \in \mathbb{C}\left[p_{1}\left(x_{1}, \ldots, x_{n}\right), \ldots, p_{n}\left(x_{1}, \ldots, x_{n}\right)\right]$ as a polynomial in $p_{1}\left(x_{1}, \ldots, x_{n}\right), \ldots, p_{n}\left(x_{1}, \ldots, x_{n}\right)$ for $k>n$.

The expression of $p_{k}\left(x_{1}, \ldots, x_{n}\right)$ for all $k \in \mathbb{N}$ in the quotient ring

$$
\Lambda_{n}(x) /\left(p_{1}\left(x_{1}, \ldots, x_{n}\right)\right) \simeq \mathbb{C}\left[p_{2}\left(x_{1}, \ldots, x_{n}\right), \ldots, p_{n}\left(x_{1}, \ldots, x_{n}\right)\right]
$$

easily follows from (A.23). If we restrict to small values of $n$, we obtain the following identities.

1) If $n=1$, then we have

$$
p_{k}\left(x_{1}\right)=0
$$

for $k \geq 1$ in $\Lambda_{1}(x) /\left(p_{1}\left(x_{1}\right)\right)$.

2) If $n=2$, then we have

$$
p_{k}\left(x_{1}, x_{2}\right)= \begin{cases}2^{1-\frac{k}{2}}\left(p_{2}\left(x_{1}, x_{2}\right)\right)^{\frac{k}{2}} & \text { for } k \geq 1 \text { even } \\ 0 & \text { for } k \geq 1 \text { odd }\end{cases}
$$

in $\Lambda_{2}(x) /\left(p_{1}\left(x_{1}, x_{2}\right)\right)$.

3) If $n=3$, then we have

$$
p_{k}\left(x_{1}, x_{2}, x_{3}\right)=\sum_{\substack{2 r_{2}+3 r_{3}=k \\
r_{2}, r_{3} \in \mathbb{N}_{0}}} \frac{2 r_{2}+3 r_{3}}{r_{2}+r_{3}}\left(\begin{array}{c}
r_{2}+r_{3} \\
r_{2}
\end{array}\right) \frac{\left(p_{2}\left(x_{1}, x_{2}, x_{3}\right)\right)^{r_{2}}\left(p_{3}\left(x_{1}, x_{2}, x_{3}\right)\right)^{r_{3}}}{2^{r_{2}} 3^{r_{3}}}
$$

for $k \geq 1$ in $\Lambda_{3}(x) /\left(p_{1}\left(x_{1}, x_{2}, x_{3}\right)\right)$. 


\section{A.3 The Cauchy kernel}

Let $x=\left(x_{1}, x_{2}, \ldots\right)$ and $y=\left(y_{1}, y_{2}, \ldots,\right)$ be two infinite sequences of independent variables. We shall review the series expansions for the infinite product

$$
\Omega^{*}=\prod_{i, j \in \mathbb{N}}\left(1+x_{i} y_{j}\right)
$$

called the dual Cauchy kernel.

Proposition A.1. We have

$$
\prod_{i, j \in \mathbb{N}}\left(1+x_{i} y_{j}\right)=\sum_{\lambda \in \mathcal{P}} e_{\lambda}(x) m_{\lambda}(y)=\exp \left(\sum_{n=1}^{\infty} \frac{(-1)^{n+1}}{n} p_{n}(x) p_{n}(y)\right)=\sum_{\lambda \in \mathcal{P}} \varepsilon_{\lambda} \frac{p_{\lambda}(x) p_{\lambda}(y)}{z_{\lambda}} .
$$

Proof. For the first identity in (A.29), we may write

$$
\prod_{i, j \in \mathbb{N}}\left(1+x_{i} y_{j}\right)=\prod_{j=1}^{\infty}\left(\prod_{i=1}^{\infty}\left(1+x_{i} y_{j}\right)\right)=\prod_{j=1}^{\infty} \sum_{k=0}^{\infty} e_{k}(x) y_{j}^{k}=\sum_{\lambda \in \mathcal{P}} e_{\lambda}(x) m_{\lambda}(y) .
$$

For the second identity in (A.29), let us recall the power series expansions of

$$
\log (1+z)=\sum_{n=1}^{\infty}(-1)^{n+1} \frac{z^{n}}{n} \quad \text { and } \quad \exp (z)=\sum_{n=0}^{\infty} \frac{z^{n}}{n !}
$$

Therefore, we have

$$
\begin{aligned}
\log \prod_{i, j \in \mathbb{N}}\left(1+x_{i} y_{j}\right) & =\sum_{i, j \in \mathbb{N}} \log \left(1+x_{i} y_{j}\right)=\sum_{i, j \in \mathbb{N}} \sum_{n=1}^{\infty}(-1)^{n+1} \frac{\left(x_{i} y_{j}\right)^{n}}{n} \\
& =\sum_{n=1}^{\infty} \frac{(-1)^{n+1}}{n}\left(\sum_{i=1}^{\infty} x_{i}^{n}\right)\left(\sum_{j=1}^{\infty} y_{j}^{n}\right)=\sum_{n=1}^{\infty} \frac{(-1)^{n+1}}{n} p_{n}(x) p_{n}(y),
\end{aligned}
$$

which implies

$$
\begin{aligned}
\prod_{i, j \in \mathbb{N}}\left(1+x_{i} y_{j}\right) & =\exp \left(\sum_{n=1}^{\infty} \frac{(-1)^{n+1}}{n} p_{n}(x) p_{n}(y)\right)=\sum_{k=0}^{\infty} \frac{1}{k !}\left(\sum_{n=1}^{\infty} \frac{(-1)^{n+1}}{n} p_{n}(x) p_{n}(y)\right)^{k} \\
& =\sum_{k=0}^{\infty} \frac{1}{k !}\left(\sum_{r_{1}, r_{2}, \cdots \in \mathbb{N}_{0}}\left(\begin{array}{c}
k \\
r_{1}, r_{2}, \ldots
\end{array}\right)\left(\frac{p_{1}(x) p_{1}(y)}{1}\right)^{r_{1}}\left(-\frac{p_{2}(x) p_{2}(y)}{2}\right)^{r_{2}} \ldots\right) \\
& =\sum_{\lambda \in \mathcal{P}} \varepsilon_{\lambda} \frac{p_{\lambda}(x) p_{\lambda}(y)}{z_{\lambda}},
\end{aligned}
$$

where $\left(\begin{array}{c}k \\ r_{1}, r_{2}, \ldots\end{array}\right)=\frac{k !}{r_{1} ! r_{2} ! \ldots}$ for $r_{1}+r_{2}+\cdots=k$ denotes the multinomial coefficient. This completes the proof.

\section{Conclusion and outlook}

As we already noticed, conformal Galilei algebras $\mathfrak{c} g a_{1}(d, \mathbb{R})$ are given by the Inönu-Wigner contraction of conformal Lie algebras $\mathfrak{s o}(d+1,2, \mathbb{R})$. Homomorphisms of generalized Verma modules for conformal Lie algebras were already classified, see e.g. [9], so it would be desirable to understand the process of contraction directly on the level of homomorphisms of (generalized) Verma modules (or singular vectors). 
Secondly, the singular vectors constructed in the present article are in bijective correspondence with differential operators equivariant for a conformal Galilei algebra and acting on smooth sections of line bundles supported on the homogeneous space $G / B$. The explicit form of singular vectors allows to deduce the formulas for equivariant differential operators, acting in the non-compact picture of induced representations.

We also remark that the representation of $\mathfrak{s l}(2, \mathbb{C})$ on the vector space $F$, defined in Section 3 , can be extended to the so called Fock representation of the Virasoro algebra, cf. [13.

\section{Acknowledgement}

L. Křižka and P. Somberg acknowledge the financial support from the grant GA CR P201/12/G028.

\section{References}

[1] Naruhiko Aizawa, Radhakrishnan Chandrashekar, and Jambulingam Segar, Lowest weight representations, singular vectors and invariant equations for a class of conformal Galilei algebras, SIGMA 11 (2015), 002, 19 pages.

[2] Naruhiko Aizawa, Vladimir K. Dobrev, and Heinz-Dietrich Doebner, Intertwining operators for Schrödinger algebras and hierarchy of invariant equations, Quantum theory and symmetries (Kraków, 2001), World Sci. Publ., River Edge, NJ, 2002, pp. 222-227.

[3] Naruhiko Aizawa and Phillip S. Isaac Yuta Kimura, Highest weight representations and Kac determinants for a class of conformal Galilei algebras with central extension, Int. J. Math. 23 (2012), no. 10, 1250118, 25 pages.

[4] Naruhiko Aizawa, Yuta Kimura, and Jambulingam Segar, Intertwining operators for $\ell$ conformal Galilei algebras and hierarchy of invariant equations, J. Phys. A 46 (2013), no. 40, 405204, 14 pages.

[5] Arjun Bagchi and Rajesh Gopakumar, Galilean conformal algebras and AdS/CFT, J. High Energy Phys. 2009 (2009), no. 7, 037, 22 pages.

[6] Peter Havas and Jerzy F. Plebański, Conformal extensions of the Galilei group and their relation to the Schrödinger group, J. Math. Phys 19 (1978), no. 2, 482-488.

[7] Nathan Jacobson, Lie algebras, Dover Publications, New York, 1979.

[8] Libor Křrižka and Petr Somberg, Algebraic analysis on scalar generalized Verma modules of Heisenberg parabolic type I.: $A_{n}$-series, arXiv:1502.07095 (2015), (accepted to Transformation Groups).

[9] Toshiyuki Kobayashi, Bent Oersted, Petr Somberg, and Vladimír Souček, Branching laws for Verma modules and applications in parabolic geometry I., Adv. Math. 285 (2015), 1-57.

[10] Rencai Lü, Volodymyr Mazorchuk, and Kaiming Zhao, On simple modules over conformal Galilei algebras, J. Pure Appl. Algebra 218 (2014), no. 10, 1885-1899.

[11] Ian R. Macdonald, Symmetric functions and Hall polynomials, Oxford Mathematical Monographs, Oxford University Press, New York, 1995.

[12] Dario Martelli and Yuji Tachikawa, Comments on Galilean conformal field theories and their geometric realization, J. High Energy Phys. 2010 (2010), no. 5, 091, 31 pages.

[13] Minoru Wakimoto and Hirofumi Yamada, The Fock representations of the Virasoro algebra and the Hirota equations of the modified KP hierarchies, Hiroshima Math. J. 16 (1986), no. 2, 427-441. 
[14] Xiaoping $\mathrm{Xu}$, Flag partial differential equations and representations of Lie algebras, Acta Appl. Math. 102 (2008), no. 2-3, 249-280.

[15] — Algebraic approaches to partial differential equations, Springer, Heidelberg, 2013.

(L. Křižka) Charles University in Prague, Faculty of Mathematics and Physics, Mathematical Institute of Charles University, Sokolovská 83, 18000 Praha 8, Czech Republic

E-mail address: krizka@karlin.mff.cuni.cz

(P.Somberg) Charles University in Prague, Faculty of Mathematics and Physics, Mathematical Institute of Charles University, Sokolovská 83, 18000 Praha 8, Czech Republic

E-mail address: somberg@karlin.mff.cuni.cz 